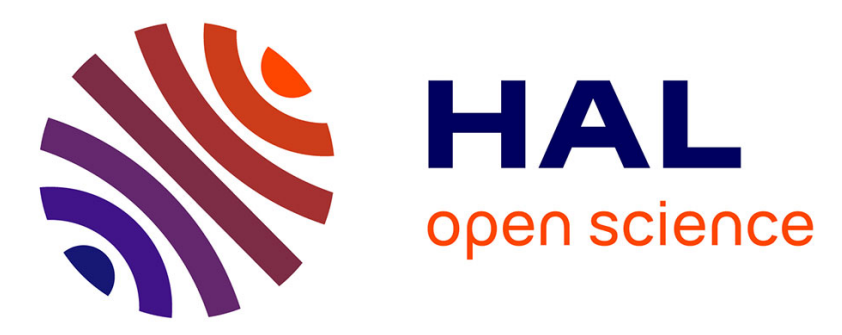

\title{
Time-domain damping models in structural acoustics using digital filtering
}

\author{
Augustin Parret-Fréaud, Benjamin Cotté, Antoine Chaigne
}

\section{To cite this version:}

Augustin Parret-Fréaud, Benjamin Cotté, Antoine Chaigne. Time-domain damping models in structural acoustics using digital filtering. Mechanical Systems and Signal Processing, 2016, 68-69, pp.587607. 10.1016/j.ymssp.2015.08.005 . hal-01253180

\section{HAL Id: hal-01253180 \\ https://hal-ensta-paris.archives-ouvertes.fr/hal-01253180}

Submitted on 24 Apr 2018

HAL is a multi-disciplinary open access archive for the deposit and dissemination of scientific research documents, whether they are published or not. The documents may come from teaching and research institutions in France or abroad, or from public or private research centers.
L'archive ouverte pluridisciplinaire HAL, est destinée au dépôt et à la diffusion de documents scientifiques de niveau recherche, publiés ou non, émanant des établissements d'enseignement et de recherche français ou étrangers, des laboratoires publics ou privés. 


\title{
Time-domain damping models in structural acoustics using digital filtering
}

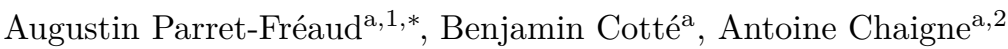 \\ ${ }^{a}$ Department of Mechanical Engineering (UME), ENSTA ParisTech \\ 828 boulevard des Maréchaux, 91762 Palaiseau Cedex, France
}

\begin{abstract}
This paper describes a new approach in order to formulate well-posed timedomain damping models able to represent various frequency domain profiles of damping properties. The novelty of this approach is to represent the behavior law of a given material directly in a discrete-time framework as a digital filter, which is synthesized for each material from a discrete set of frequency-domain data such as complex modulus through an optimization process. A key point is the addition of specific constraints to this process in order to guarantee stability, causality and verification of thermodynamics second law when transposing the resulting discrete-time behavior law into the time domain. Thus, this method offers a framework which is particularly suitable for time-domain simulations in structural dynamics and acoustics for a wide range of materials (polymers, wood, foam...), allowing to control and even reduce the distortion effects induced by time-discretization schemes on the frequency response of continuous-time behavior laws.
\end{abstract}

Keywords: Damping, time-domain modeling, vibroacoustics, digital filtering.

\section{Introduction}

Damping plays a major role in vibration of structures because of its huge influence on various characteristics of dynamical response and radiated acoustic

\footnotetext{
Corresponding author

Email addresses: augustin.parret-freaud@safran.fr (Augustin Parret-Fréaud), benjamin.cotte@ensta-paristech.fr (Benjamin Cotté), chaigne@mdw.ac.at (Antoine Chaigne)

${ }^{1}$ Present address: Safran Tech, 1 rue Geneviève Aubé, 78772 Magny les Hameaux, France.

${ }^{2}$ Present address: Institut für Wiener Klangstil, University of Music and Performing Arts Vienna (MDW), Anton-von-Webern Platz 1, 1030 Vienna, Austria.
} 
field $[1,2]$. A good understanding of this phenomenon is of importance to 5 achieve multiple goals ranging from the attenuation of noise and vibrations [3] to the improvement of efficiency regarding radiated sound fields, e.g. in audio transducers or musical instruments. In this context, the study and prediction of transient vibroacoustic response generated by structures under impulse-type excitation is a major field of interest which requires a fine description of damping.

10 One may find various applications in transportation acoustics, e.g. in railways industry when studying impact noise generated by wheel-rail contact [4] or in building and room acoustics through the concept of auralization [5]. Another major area of application is related to the concept of sound synthesis by means of physical modeling [6] and its applications in the acoustics of musical instruments 15 [7-9], where psychoacoustical studies have highlighted the major role played by damping on the perception of sounds [10].

For a given material, damping is often characterized in the frequency domain from experimental measurement of the complex modulus $E^{*}$ or loss factor $\eta$, that may be obtained using various techniques including viscoanalysers. Such results 20 generally exhibit frequency behaviors which differ a lot depending on the type of observed material and on external conditions like temperature and hygrometry (see e.g. some experimental results for a wide range of damping materials in [11]). This leads to a wide range of frequency-domain damping models (see e.g. [12]), which may not be directly transferable to the time domain.

On the other hand, time-domain formalism appears to be more natural when dealing with structures excited by impulses as it naturally takes transient aspects of simulated response into account, or in presence of nonlinear vibrations. In this framework, the literature is plentiful of models initially developed in the framework of linear viscoelasticity [13]. The most simple ones are well-known Maxwell, Kelvin-Voigt and Zener (also called Standard Linear Solid) models, whereas more sophisticated ones has been also developed, such as Golla-Huges-McTavish [14, 15], Anelastic Displacement Fields [16] or generalized Zener model, which is probably the most widely used in linear viscoelasticity $[17,18]$. The common basis of all those models is to describe damping behav35 ior by combining rheological elements, such as springs, dash-pots and masses, which are expressed in the time domain by combination of time derivatives. 
However, their ability to approach arbitrary frequency-domain variations of a given behavior may be limited and they may require, for special shapes, a large number of coefficients. Another class of model that has been widely studied 40 for about twenty years lies on the concept of fractional time derivatives [19]. Their main advantage lies in the ability to represent almost constant behavior in the frequency domain using only a small set of coefficients, together with being well-posed, especially concerning causality and thermodynamics aspects [20]. However, an important drawback of such types of models remains in the difficulty to transpose it into a numerical time-integration scheme in an efficient way [21], and current implementations often requires a significant number of internal variables.

Hence, providing time-domain models able to accurately describe various frequency shapes of damping properties while being in accordance with essential 50 properties of causality, stability and positivity of dissipation remains a sensitive issue that has been recently addressed, for linear viscoelasticity, in an original way using existing mathematical works on complex analysis [22]. Moreover, in the context of numerical simulation, time-discretization schemes applied to a given continuous time-domain model have to be chosen accordingly since they 55 often lead to distortion of its response in the frequency domain, especially in the frequency range close to the Nyquist frequency $f_{s} / 2$ which is the maximum valid frequency associated to a sampling rate $f_{s}$ according to Shannon theorem. Furthermore, it is desirable to make sure that the whole numerical scheme resulting from space-time discretization of the initial problem fulfills some discrete-time energetic identity, especially in order to guarantee the stability of the resolution [6].

The work presented here lies in the three axes mentioned above as our goal is to provide time-domain models able to depict various types of frequency dependency for damping properties, so as to be usable in the context of time-domain simulation of structures made from a wide range of materials (including wood, polymers and composites) under impulse loading. In order to take the influence of the discretization step into account, the key point of the approach described in the present paper is to directly work inside a discrete-time framework by considering the behavior law as a digital filter [23]. Given a sampling frequency 
corresponding to the time step of the simulation, the filter is then synthesized in the frequency domain from experimental results on complex modulus through an optimization problem involving the discrete transfer function of the filter. Essential properties as stability, causality and positivity of dissipation are ensured during the optimization process through the addition of specific constraints acting on filter coefficients. Once synthesized, the resulting filter, which corresponds to an already discretized behavior law, may be directly transposed in the discrete-time domain keeping its well-posedness. Finally, one obtains a recursive relation between values at current and previous time steps, which may be directly implemented in a discrete integration scheme, providing discrete-time so energetic identity of the whole scheme as well.

This paper is organized as follows. On the basis of a one-dimensional problem that will remain the application framework throughout the paper, Section 2 introduces the formulation of discrete well-posed time-domain behavior law by means of digital filtering. Section 3 shows the constrained optimization process used to synthesize filters from experimental frequency-domain data. Section 4 describes the space-time numerical scheme to simulate structures equipped with previous models, followed by stability analysis by means of an energetic approach and study of errors induced by numerical dispersion and dissipation. Section 5 presents numerical assessments that (1) describes the whole pro90 cess of modeling and time-domain simulation of a structure given experimental frequency-domain results and (2) validate the whole approach, especially regarding numerical errors.

\section{Time domain modeling by means of digital filtering}

\subsection{Model problem}

The present development, mainly those regarding the integration scheme, is realized in the context of one-dimensional mechanical problems. To present the method in a simple context, we consider below the longitudinal vibrations of a cantilever beam under the classical small perturbations hypothesis (Fig. 1).

Denoting $u(x, t), \varepsilon(x, t)$ and $\sigma(x, t)$ the displacement, strain and stress fields, respectively, and applying Newton second law, leads to the following system of 


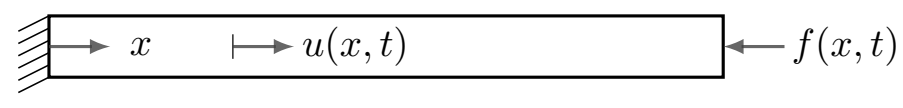

Figure 1: Cantilever beam schematics.

equations:

$$
\begin{aligned}
\sigma(x, t) & =\mathcal{E}(\varepsilon(x, t))=\mathcal{E}\left(\frac{\partial u(x, t)}{\partial x}\right), \\
\rho(x) \frac{\partial^{2} u(x, t)}{\partial t^{2}} & =\frac{\partial \sigma(x, t)}{\partial x}+f(x, t),
\end{aligned}
$$

where the constitutive law $\mathcal{E}$ is assumed to be local, linear and time-invariant,

In accordance with the hypothesis of linearity and time-invariance on $\mathcal{E}$, one can express the filter $\mathcal{H}$ in the time domain as a linear recursive relation involving values of $\sigma$ and $\varepsilon$ at current and previous time steps [23]:

$$
\begin{aligned}
\sigma^{n+1}(x) & =\mathcal{H}\left(\sigma^{n}(x), \ldots, \sigma^{n+1-N_{d}}(x), \varepsilon^{n+1}(x), \ldots, \varepsilon^{n+1-N_{c}}(x)\right) \\
& =H_{0}\left(\varepsilon^{n+1}(x)+\sum_{l=1}^{N_{c}} c_{l} \varepsilon^{n+1-l}(x)\right)-\sum_{m=1}^{N_{d}} d_{m} \sigma^{n+1-m}(x) .
\end{aligned}
$$

where $H_{0},\left\{c_{l}\right\}_{l}$ and $\left\{d_{m}\right\}_{m}$ denote time-invariant coefficients. 
The z-transform (denoted TZ) provides a convenient way to synthetize the filter $\mathcal{H}$ and study its properties in the frequency domain. Given a sequence of discrete samples $\left\{v^{n}\right\}_{n}$ at different time $t^{n}$, the corresponding z-transform $\check{v}(z)$ is given by:

$$
\mathrm{TZ}:\left\{v^{n}\right\}_{n} \longmapsto \check{v}(z)=\sum_{n=-\infty}^{\infty} v^{n} z^{-n} .
$$

Applying TZ to the discrete-time constitutive relation (2) gives the complex z-transfer function $H(z)$ as:

$$
H(z)=\frac{\check{\sigma}(x, z)}{\check{\varepsilon}(x, z)}=H_{0}\left[\frac{1+\sum_{l=1}^{N_{c}} c_{l} z^{-l}}{1+\sum_{m=1}^{N_{d}} d_{m} z^{-m}}\right],
$$

where $\check{\sigma}(x, z)($ resp. $\check{\varepsilon}(x, z))$ is the Z-transform of $\left\{\sigma^{n}(x)\right\}_{n}\left(\operatorname{resp} .\left\{\varepsilon^{n}(x)\right\}_{n}\right)$. The transfer function $H$ may be equally written in a form involving its poles $\left(p_{m}\right)_{1 \leq m \leq N_{d}}$ and zeros $\left(q_{l}\right)_{1 \leq l \leq N_{c}}$ as:

$$
H(z)=H_{0}\left[\frac{\prod_{l=1}^{N_{c}}\left(1-q_{l} z^{-l}\right)}{\prod_{m=1}^{N_{d}}\left(1-p_{m} z^{-m}\right)}\right] .
$$

Let us note that this last expression is particularly interesting as many properties of digital filters derive from conditions on the poles and zeros of their transfer functions. Within the scope of the present study, the very first properties to fulfill are the stability and causality of the digital filter $\mathcal{H}$. Those properties are satisfied by the necessary and sufficient condition on the poles $\left(p_{m}\right)_{1 \leq m \leq N_{d}}$ of the transfer function which have to remain strictly inside the unit circle (see e.g. $[24])$ :

$$
\left|p_{m}\right|<1, \quad 1 \leq m \leq N_{d}
$$

In the following, this last condition will be the first mandatory constraint imposed to any synthesis process of $\mathcal{H}$.

For the rest of the paper, instead of working with the general expression (5), we restrict ourselves to the class of transfer functions $H$ resulting from the sum of $N_{f}$ one-pole sub-filter $H_{k}$ together with a constant function (pure gain) $H_{0}$ :

$$
H(z)=H_{0}+\sum_{k=1}^{N_{f}} H_{k}(z)=H_{0}+\sum_{k=1}^{N_{f}} \frac{H_{0 k}}{1-p_{k} z^{-1}} .
$$

The previous expression is actually the partial fraction expansion of every trans115 fer function (5) satisfying $N_{c}=N_{d}=N_{f}$ and having poles of unit multiplicity, 
which restricts our scope to the discrete models which exclude elementary filters with multiple or complex conjugate poles. This fundamental assumption has been done to simplify the energy-based stability analysis performed in Section 4.2. Also, let us note that using a parallel association process with poles of unit multiplicy is a common approach in continuous-domain identification (e.g. generalized Zener model) which seems to perform well for a wide class of viscoelastic materials. Besides, from a practical point of view, the optimization algorithm used in Section 3 in order to synthetize filter $H$ from continuous frequency-domain data allows the poles of the transfer function to be as close as possible if needed.

\subsection{Properties of one-pole filters $H_{k}$ in the continuous frequency domain}

Before going further into details about the synthesis of filter $H$ based on experimental complex modulus, we will describe here the main properties of the continuous frequency response $H_{k}^{c}(\omega)$ (where $\omega$ denotes the angular frequency) of one-pole elementary filters $H_{k}(z)$ when poles $p_{k}$ satisfy condition (6). Such a response is usually obtained by evaluating $H_{k}(z)$ on the unit circle by means of the change of variable $z \leftarrow \exp \left(i \omega f_{s}^{-1}\right)$ :

$$
H_{k}^{c}(\omega)=H_{k, r}^{c}(\omega)+i H_{k, i}^{c}(\omega) \quad\left(\omega \in\left[0, \pi f_{s}^{-1}\right]\right),
$$

with:

$$
\begin{aligned}
H_{k, r}^{c}(\omega) & =H_{0, k} \frac{1-p_{k} \cos \left(\omega f_{s}^{-1}\right)}{1+p_{k}^{2}-2 p_{k} \cos \left(\omega f_{s}^{-1}\right)}, \\
H_{k, i}^{c}(\omega) & =-H_{0, k} \frac{p_{k} \sin \left(\omega f_{s}^{-1}\right)}{1+p_{k}^{2}-2 p_{k} \cos \left(\omega f_{s}^{-1}\right)} .
\end{aligned}
$$

The derivation of $H_{k, r}^{c}$ with respect to $\omega$ gives:

$$
\frac{d H_{k, r}^{c}}{d \omega}(\omega)=-H_{0, k} p_{k} \frac{\left(1-p_{k}^{2}\right) f_{s}^{-1} \sin \left(\omega f_{s}^{-1}\right)}{\left(1+p_{k}^{2}-2 p_{k} \cos \left(\omega f_{s}^{-1}\right)\right)^{2}},
$$

such that when hypothesis (6) on $p_{k}$ is verified, $H_{k, r}^{c}$ is a monotonously increasing function if $H_{0, k} p_{k} \leq 0$, with the following extrema:

$$
\begin{aligned}
H_{k, r_{\text {min }}}^{c} & =H_{k, r}^{c}(0)=\frac{H_{0, k}}{1-p_{k}}, \\
H_{k, r_{\text {max }}}^{c} & =H_{k, r}^{c}\left(\pi f_{s}\right)=\frac{H_{0, k}}{1+p_{k}}, \\
\left|\Delta H_{k, r}^{c}\right| & =\left|H_{k, r_{\text {max }}}^{c}-H_{k, r_{\text {min }}}^{c}\right|=\frac{2\left|H_{0, k} p_{k}\right|}{1-p_{k}^{2}} .
\end{aligned}
$$


The same applied to $H_{k, i}^{c}$ gives:

$$
\frac{d H_{k, i}^{c}}{d \omega}(\omega)=H_{0, k} p_{k} f_{s}^{-1} \frac{\left(2 p_{k}-\left(1+p_{k}^{2}\right) \cos \left(\omega f_{s}^{-1}\right)\right)}{\left(1+p_{k}^{2}-2 p_{k} \cos \left(\omega f_{s}^{-1}\right)\right)^{2}},
$$

such that $H_{k, i}^{c}$ vanishes at $\omega=0$ and $\omega=\pi f_{s}$ and admits a unique extremum $H_{k, i}^{c}$ max $\omega_{\max }^{H_{k, i}^{c}}$ given by:

$$
H_{k, i_{\max }}^{c}=-\frac{H_{0, k} p_{k}}{1-p_{k}^{2}}, \quad \omega_{\max }^{H_{k, i}^{c}}=f_{s} \arccos \left(\frac{2 p_{k}}{1+p_{k}^{2}}\right) .
$$

Thus, $H_{k, i}^{c}(\omega) \geq 0$ as soon as $H_{0, k} p_{k} \leq 0$ and (6) is satisfied. Finally, Figure 3 shows the response $H_{k, r}^{c}$ and $H_{k, i}^{c}$ of filter $H_{k}$ for different values of $p_{k}$ satisfying (6).
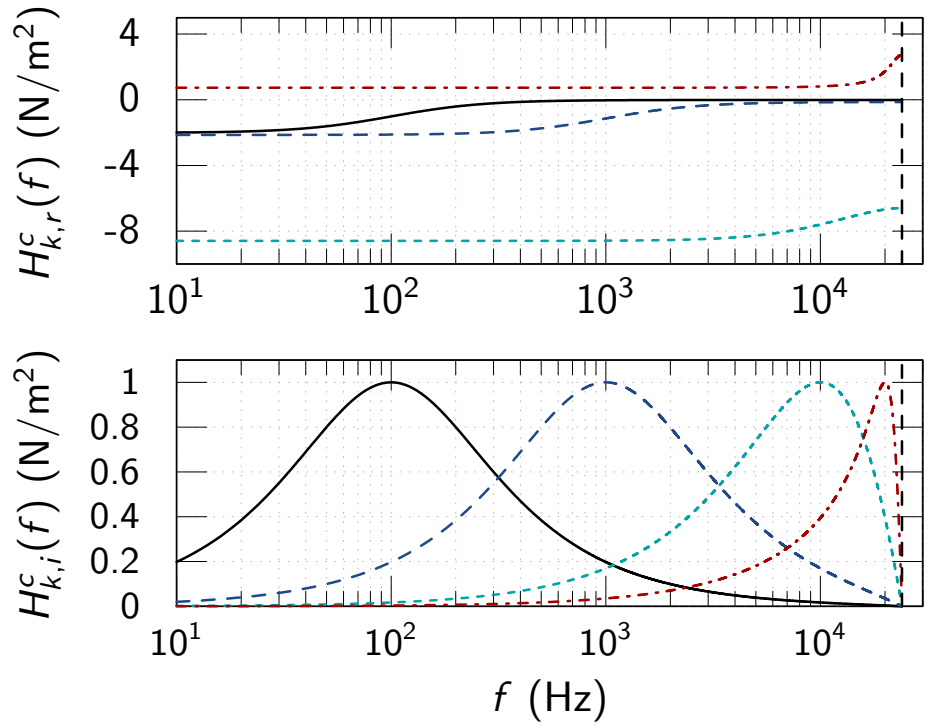

Figure 3: $H_{k, r}^{c}$ and $H_{k, i}^{c}$ for values of $p_{k}$ satisfying (6) and $H_{0 k} p_{k} \leq 0\left(f_{s}=48 \mathrm{kHz}\right)$ : $p_{k}=0.99(-), p_{k}=0.88(--), p_{k}=0.13(--)$ and $p_{k}=-0.58(-\cdot)$. The vertical dashed line corresponds to $f_{s} / 2$.

Let us now consider the continuous frequency response $H^{c}(\omega)$ of the filter $H$ given by (7), which can be written:

$$
H^{c}(\omega)=H_{r}^{c}(\omega)+i H_{i}^{c}(\omega) \quad\left(\omega \in\left[0, \pi f_{s}^{-1}\right]\right)
$$


with:

$$
\begin{aligned}
& H_{r}^{c}(\omega)=H_{0}+\sum_{k=1}^{N_{f}} H_{0, k} \frac{1-p_{k} \cos \left(\omega f_{s}^{-1}\right)}{1+p_{k}^{2}-2 p_{k} \cos \left(\omega f_{s}^{-1}\right)}, \\
& H_{i}^{c}(\omega)=-\sum_{k=1}^{N_{f}} H_{0, k} \frac{p_{k} \sin \left(\omega f_{s}^{-1}\right)}{1+p_{k}^{2}-2 p_{k} \cos \left(\omega f_{s}^{-1}\right)} .
\end{aligned}
$$

Taking the continuous frequency-domain transposition of equation (4), one has:

$$
H^{c}(\omega)=\frac{\hat{\sigma}(x, \omega)}{\hat{\varepsilon}(x, \omega)}
$$

where $\hat{v}(x, \omega)$ stands for the partial time Fourier transform of $v(x, t)$ given for causal signals by:

$$
\hat{v}(x, \omega)=\int_{0}^{\infty} v(x, t) \exp (-i \omega t) d t .
$$

From a mechanical point of view, Equation (14) defines the usual complex modulus of a given material as the ratio of the Fourier transform of stress and strain. Thus, we may interpret $H^{c}(\omega)$ as the complex modulus in the continuous frequency-domain associated with a discrete-time behavior law defined by filter $H$. In order to obtain a well-posed model, the first additional condition to impose is the positivity of the static modulus as:

$$
H^{c}(0)=H(1) \geq 0 \text {. }
$$

Furthermore, the global loss factor $\eta^{H}$ and elementary ones $\eta_{k}^{H}$ associated to elementary one-pole filters may be introduced in a similar way as for the complex modulus:

$$
\begin{array}{r}
H^{c}(\omega)=H_{r}^{c}(\omega)\left(1+i \eta^{H}(\omega)\right)=H_{r}^{c}(\omega)\left(1+i \sum_{k=1}^{N_{f}} \eta_{k}^{H}(\omega)\right) \\
\text { with } \eta_{k}^{H}(\omega)=\frac{H_{k, i}^{c}(\omega)}{H_{r}^{c}(\omega)} .
\end{array}
$$

In order to obtain a dissipative model in accordance with the second law of thermodynamics, we choose to impose the positivity of each loss factor $\eta_{k}^{H}$, so as to ensure each internal process act as a dissipative one. From Equations (9b) and (13a), this may be enforced by the following condition acting on the coefficients of $H_{k}$ :

$$
H_{0, k} p_{k} \leq 0, \quad \forall k,
$$


which ensure functions $H_{i, k}^{c}$ to be positive and $H_{r, k}^{c}$ to be monotonously increasing, as already seen in Section 2.3. This last property, together with condition (16) on poles $p_{k}$, ensure positivity of $H_{r}^{c}$, and thus of each function $\eta_{k}^{H}$ on the whole frequency range. Let us note that condition (18) is only a sufficient one to obtain a positive global loss factor $\eta^{H}$ and the dissipation property of the underlying behavior. Indeed, we may exhibit filters constituted of some components with negative dissipation, which may be balanced by the overall dissipation induced by the remaining ones so that the global loss factor $\eta^{H}$ remains positive on the whole frequency range. As one must be careful when dismissing a model which is itself physical only because some of its elementary components appear to be not physical, it would be probably interesting to try to relax this constraint in future works even if it might complicate the whole synthesis process.

\section{Synthesis of filter $\mathcal{H}$ from frequency-domain damping data}

Given a set $\left\{E_{j}^{*}\right\}_{j}$ of values of Young's modulus at various frequencies $\left\{\omega_{j}\right\}_{j}$, which may be results of experimental procedures, this section is devoted to the description of the optimization process that allows us to synthesize the filter $H(z)$ previously introduced. The underlying problem consists in finding parameters $H_{0},\left\{H_{0, k}, p_{k}\right\}_{k}$ minimizing the distance between the continuous response $H^{c}(\omega)$ of filter $\mathcal{H}$ and the value $E_{j}^{*}$ at each frequency $\omega_{j}$, while ensuring conditions (6), (16) and (18) in order to guarantee the well-posedness of the corresponding discrete-time model. From a general point of view, finding an efficient method to correctly identify a material law from data given in the complex plane is still a tricky task, though it has been recently addressed in some original way when identifying rheological behaviors, using mathematical results from complex analysis [22] or graphical methods stemming from the field of system automation [25].

\subsection{Cost function and constrained optimization problem}

In the following, we choose to work with a non-linear least-square problem by directly measuring the distance between $H^{c}$ and the input data using the classical quadratic norm on both real and imaginary part. Denoting 
$X=\left[H_{0},\left\{H_{0, k}, p_{k}\right\}_{k}\right]^{T}$ the vector containing filter parameters, the resulting cost function $\mathcal{L}$ to be minimized is:

$$
\begin{aligned}
\mathcal{L}(X)= & \alpha_{r} \mathcal{L}_{r}(X)+\alpha_{i} \mathcal{L}_{i}(X), \\
= & \alpha_{r}\left[\sum_{j}\left(\frac{H_{r}^{c}\left(\omega_{j}\right)-\mathcal{R} e\left(E_{j}^{*}\right)}{\mathcal{R} e\left(E_{j}^{*}\right)}\right)^{2}\right]^{\frac{1}{2}} \\
& +\alpha_{i}\left[\sum_{j}\left(\frac{H_{i}^{c}\left(\omega_{j}\right)-\mathcal{I} m\left(E_{j}^{*}\right)}{\mathcal{I} m\left(E_{j}^{*}\right)}\right)^{2}\right]^{\frac{1}{2}},
\end{aligned}
$$

where $\alpha_{r}$ and $\alpha_{i}$ are scaling coefficients, which will be set to 1 in the following.

In order to study the sensitivity of the cost function $\mathcal{L}$ to the parameters of the optimization process, we first express, for an arbitrary parameter $Z$, the partial derivative of $\mathcal{L}$ with respect to $Z$ as a function involving the sensitivity of $H^{c}$ :

$$
\begin{aligned}
\partial_{Z} \mathcal{L}= & \alpha_{r} \mathcal{L}_{r}{ }^{-1} \sum_{j}\left(\frac{H_{r}^{c}\left(\omega_{j}\right)-\mathcal{R} e\left(E_{j}^{*}\right)}{\mathcal{R} e\left(E_{j}^{*}\right)^{2}} \partial_{Z} H_{r}^{c}\left(\omega_{j}\right)\right) \\
& +\alpha_{i} \mathcal{L}_{i}{ }^{-1} \sum_{j}\left(\frac{H_{i}^{c}\left(\omega_{j}\right)-\mathcal{I} m\left(E_{j}^{*}\right)}{\mathcal{I} m\left(E_{j}^{*}\right)^{2}} \partial_{Z} H_{i}^{c}\left(\omega_{j}\right)\right)
\end{aligned}
$$

Then, the derivative of $H_{r}^{c}$ and $H_{i}^{c}$ with respect to $H_{0}, H_{0, k}$ and $p_{k}$ may be written from (7), (9a) and (9b) as:

$$
\begin{aligned}
\partial_{H_{0}} H_{r}^{c}(\omega) & =1 \\
\partial_{H_{0}} H_{i}^{c}(\omega) & =0 \\
\partial_{H_{0, k}} H_{r}^{c}(\omega) & =\frac{1-p_{k} \cos \left(\omega f_{s}^{-1}\right)}{1+p_{k}^{2}-2 p_{k} \cos \left(\omega f_{s}^{-1}\right)}, \\
\partial_{H_{0, k}} H_{i}^{c}(\omega) & =-\frac{p_{k} \sin \left(\omega f_{s}^{-1}\right)}{1+p_{k}^{2}-2 p_{k} \cos \left(\omega f_{s}^{-1}\right)}, \\
\partial_{p_{k}} H_{r}^{c}(\omega) & =H_{0, k} \frac{\left(1+p_{k}^{2}\right) \cos \left(\omega f_{s}^{-1}\right)-2 p_{k}}{\left(1+p_{k}^{2}-2 p_{k} \cos \left(\omega f_{s}^{-1}\right)\right)^{2}}, \\
\partial_{p_{k}} H_{i}^{c}(\omega) & =-H_{0, k} \frac{\left(1-p_{k}^{2}\right) \sin \left(\omega f_{s}^{-1}\right)}{\left(1+p_{k}^{2}-2 p_{k} \cos \left(\omega f_{s}^{-1}\right)\right)^{2}} .
\end{aligned}
$$

Previous equations show that the sensitivity of $\mathcal{L}$ to coefficients $p_{k}$, which follows directly from those of $\partial_{p_{k}} H_{r}^{c}$ and $\partial_{p_{k}} H_{i}^{c}$, is scaled by $H_{0, k}$, which is not the case concerning the sensitivity with respect to $H_{0}$ and $H_{0, k}$. Unfortunately, this may lead to a huge lack of sensitivity to the last parameters, and thus poor 
performance of the minimization algorithm as $H_{0, k}$ may be several order of magnitude above those of $p_{k}$ (typically $10^{9}$ versus 0.1 ). To overcome this issue, we apply some partial scaling to the filter parameters by scaling coefficients $H_{0}$ and $H_{0, k}$ with a reference value $\tilde{H}$ (typically $\tilde{H}=\max _{j}\left(\left|E_{j}^{*}\right|\right)$ ) prior to the optimization process.

Finally, the constrained optimization problem to be solved writes in a generic way as:

$$
\text { find } \begin{array}{r}
\tilde{X}=\left[\tilde{H}_{0},\left\{\tilde{H}_{0, k}, p_{k}\right\}_{k}\right]^{T} \text { verifying } \\
\min _{\tilde{X}} \mathcal{L}(\tilde{X}) \\
\text { subject to constraints } \mathcal{C}_{i}(\tilde{X})>0,
\end{array}
$$

where $\tilde{H}_{0}=H_{0} / \tilde{H}, \tilde{H}_{0, k}=H_{0, k} / \tilde{H}$, and function $\mathcal{C}_{i}$ contains the constraints (6), (16) and (18) on the filter coefficients:

$$
\mathcal{C}_{i}(\tilde{X})=\left[\begin{array}{c}
1-\left|p_{1}\right| \\
\vdots \\
1-\left|p_{N_{f}}\right| \\
\tilde{H}^{c}(0) \\
-\tilde{H}_{0,1} p_{1} \\
\vdots \\
-\tilde{H}_{0, N_{f}} p_{N_{f}}
\end{array}\right] .
$$

From the previous choices, we get a general non-linear optimization problem on both the cost function and the inequality constraints, which is unfortunately non convex and exhibits several local minima. In order to solve it, we use a generalpurpose algorithm devoted to non-linear optimization problems, namely the classical Sequential Quadratic Programming algorithm [26] and its implementation in the GNU/Octave programming language (provided by sqp function). In practice, this algorithm, which is in some ways close to Newton's method, solves in an iterative way a set of sub-problems defined by both quadratic approximation of the cost function and linear approximation of the constraints.

So far, let us note that the setup of a more optimal cost function, which may e.g. make the overall optimization problem more robust or take into account some external factors like noisy data, is still an open problem and will be studied in future works. 


\subsection{Application to a visco-elastic material}

We now apply the previous described approach to complex modulus data related to Polyurethane 24-8-1 foam at $20^{\circ} \mathrm{C}$, taken from AFWAL-TR-84-3089

In the following, the values of $\left[f_{\min }, f_{\max }\right]$ have been set to $[10 \mathrm{~Hz}, 25 \mathrm{kHz}]$, which covers the frequency range of the experimental data available from the report. As we are mostly interested in the frequencies in the audible range, which is approximately $[20 \mathrm{~Hz}, 20 \mathrm{kHz}]$, the critical material behavior below $10 \mathrm{~Hz}$ will not have a strong impact on our predictions. For a given iteration $p$ corresponding to the synthesis of a filter with $N_{f}=p$ one-pole sub-filters, we denote $\mathcal{L}\left(X_{p}\right)$ the associated error coming from the optimization process, that is the cost function (19) applied on the set $X_{p}$ containing the parameters of the resulting filter. Recall that $\mathcal{L}\left(X_{p}\right)$ measures the gap between the response of the synthesized filter and the input data. In order to assess the convergence of the whole iterative procedure previously described, we also introduce the relative error $e_{\mathcal{L}, p}$ measuring the variation of $\mathcal{L}\left(X_{p}\right)$ between two successive iterations, as:

$$
e_{\mathcal{L}, p}=\frac{\left|\mathcal{L}\left(X_{p-1}\right)-\mathcal{L}\left(X_{p}\right)\right|}{\left|\mathcal{L}\left(X_{p-1}\right)\right|}, \quad\left(2 \leq p \leq N_{f}\right)
$$

Figure 4 shows the complex modulus data and the continuous frequencydomain response of synthesized filters starting from $N_{f}=1$ sub-filters until the iterative procedure has converged. While the representation of discrete data 

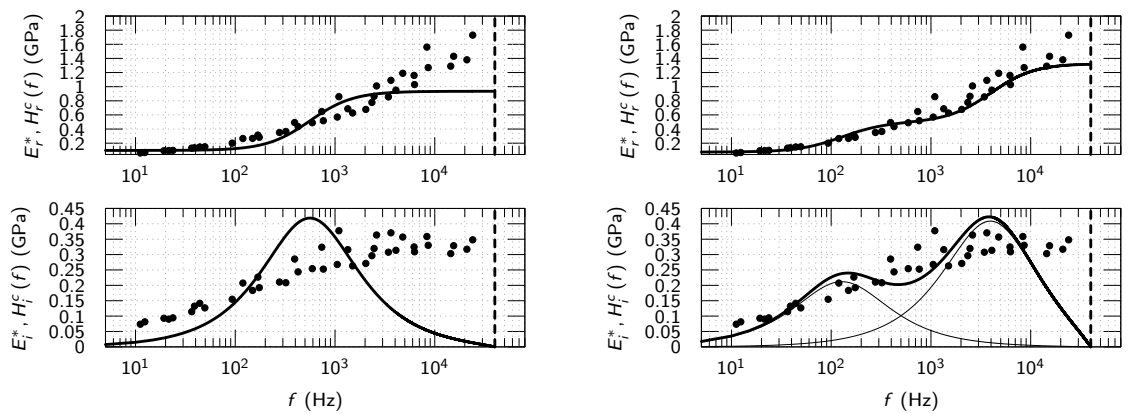

(a) $N_{f}=1, \mathcal{L}\left(X_{1}\right)=5.62$

(b) $N_{f}=2, \mathcal{L}\left(X_{2}\right)=2.81, e_{\mathcal{L}, 2}=50.1 \%$
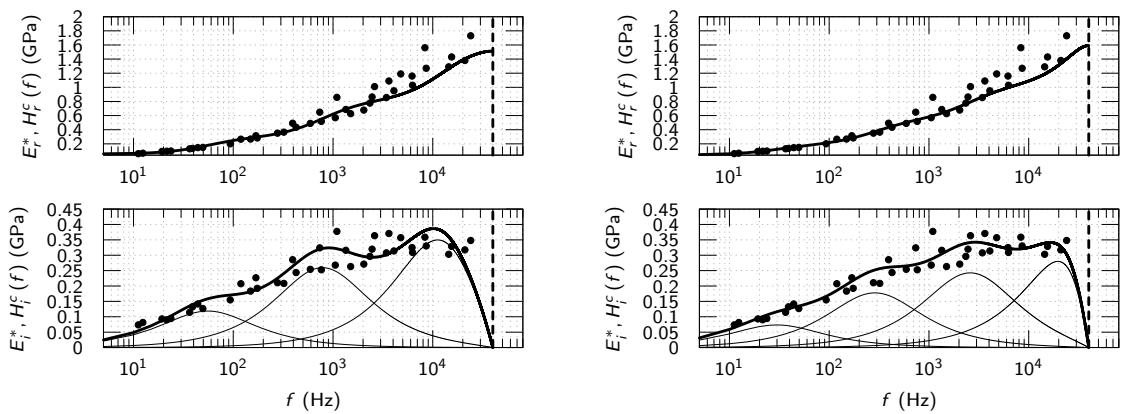

(c) $N_{f}=3, \mathcal{L}\left(X_{3}\right)=1.75, e_{\mathcal{L}, 3}=37.7 \%$

(d) $N_{f}=4, \mathcal{L}\left(X_{4}\right)=1.48, e_{\mathcal{L}, 4}=15.2 \%$
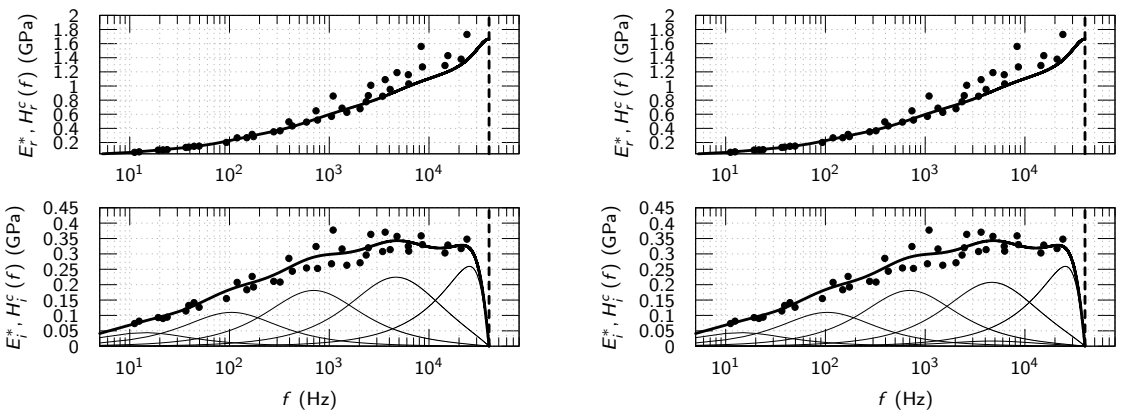

(e) $N_{f}=5, \mathcal{L}\left(X_{5}\right)=1.36, e_{\mathcal{L}, 5}=8.1 \%$

(f) $N_{f}=6, \mathcal{L}\left(X_{6}\right)=1.36, e_{\mathcal{L}, 6}=0 \%$

Figure 4: Input data (solid circles) and continuous frequency-domain response (solid curves) of synthesized filter obtained with increasing number $N_{f}$ of one-pole sub-filters. Thick curves show the response $H^{c}$ of the whole filter whereas thin curves shows the contribution $H_{k}^{c}$ of each elementary filter to the imaginary part of the response. Nyquist frequency $f_{s} / 2$ is represented by black dashed lines. 


\begin{tabular}{rl}
\hline$H_{0}, H_{0, k}$ & $p_{k}$ \\
\hline $9.8987 \cdot 10^{8}$ & \\
$-9.8006 \cdot 10^{4}$ & $9.9889 \cdot 10^{-1}$ \\
$-1.8068 \cdot 10^{6}$ & $9.9181 \cdot 10^{-1}$ \\
$-1.9877 \cdot 10^{7}$ & $9.4665 \cdot 10^{-1}$ \\
$-1.7198 \cdot 10^{8}$ & $6.8759 \cdot 10^{-1}$ \\
$1.1471 \cdot 10^{9}$ & $-2.1564 \cdot 10^{-1}$ \\
\hline
\end{tabular}

Table 1: Coefficients of synthesized filter $\mathcal{H}$ related to Polyurethane 24-8-1 for $N_{f}=5$.

keep $f_{s}$ as small as possible to save computational costs while ensuring the best representation of the initial data by the synthesized filter. 
Before concluding this section on filter synthesis, we must emphasize that the quality of the synthesized filter is highly dependent on the accuracy of the experimental values used as input, since we are generally dealing with noisy data to identify the filter parameters. Several research groups have proposed identification techniques that take into account input/output error noise, among which we can cite Mossberg et al. [27] and Pintelon et al. [28], who give complex modulus estimates together with their associated standard deviation or uncertainty bound. Another approach is followed by Collet et al. [22], who propose a noise-correction method of complex Young modulus measurements based on the three constraints of causality, positivity of the dissipation rate and reality of the relaxation function. In the future, such methods could be used to build new filter synthesis algorithms, where a confidence interval will be considered and not only exact frequency-domain data points.

\section{Time domain simulation with discrete-time constitutive relation}

The objective of the present section is to integrate previously obtained discrete-time material law into an efficient finite-difference numerical scheme, in order to solve problems defined by Equations (1).

\subsection{Explicit finite difference scheme}

We start by keeping the same time discretization (time step $\Delta t$, sampling frequency $f_{s}=\Delta t^{-1}$ ) as the one introduced in Section 2.2. In order to represent the constitutive Equation (1a), we use the discrete-time framework introduced in the previous section and transpose the transfer function (7) in the time domain, where $z^{-1}$ stands for the unit delay operator $\left(z^{-1} \mathrm{TZ}\left(v^{n}\right)=\mathrm{TZ}\left(v^{n-1}\right)\right)$. We obtain a discrete time-domain constitutive law involving $N_{f}$ internal variables $\sigma_{k}^{n}(x)$ at $t^{n}$. Furthermore, we choose to approximate equation (1b) by a leap-frog scheme, which is explicit and second order in time. Thus, the semi-discretized 
problem in time writes:

$$
\begin{aligned}
\sigma_{k}^{n+1}(x) & =H_{0 k} \frac{d u^{n+1}}{d x}(x)+p_{k} \sigma_{k}^{n}(x), k=\left\{1, \ldots, N_{f}\right\}, \\
\sigma^{n+1}(x) & =H_{0} \frac{d u^{n+1}}{d x}(x)+\sum_{k=1}^{N_{f}} \sigma_{k}^{n+1}(x), \\
\rho(x) \frac{u^{n+1}(x)-2 u^{n}(x)+u^{n-1}(x)}{\Delta t^{2}} & =\frac{d \sigma^{n}(x)}{d x}+f^{n}(x) .
\end{aligned}
$$

Regarding the spatial discretization (Figure 5), we divide the interval $[0, L]$ with $N_{h}+1$ points of equal step $h=L / N_{h}$ to obtain a first grid $\Omega_{h, 0}$ of points $x_{j}=j h$. At time $t^{n}$, we introduce the values $u_{j}^{n}$ and $f_{j}^{n}$ of $u$ and $f$ on points $x_{j}$,

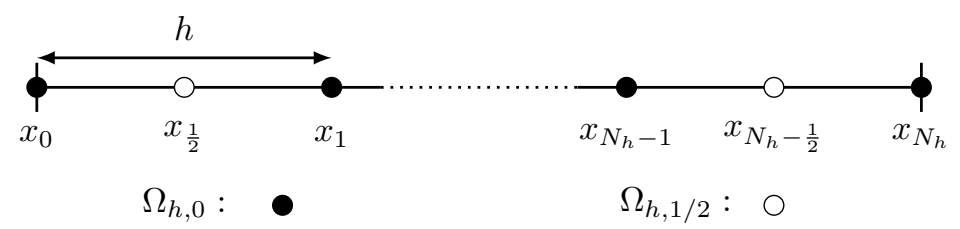

Figure 5: Spatial discretization.

and the corresponding vectors $\mathbf{u}_{h}^{n}$ and $\mathbf{f}_{h}^{n}$. We also introduce a staggered grid $\Omega_{h, 1 / 2}$ of points $x_{j+\frac{1}{2}}$ on which we define the values $\sigma_{j+\frac{1}{2}}^{n}$ and $\varepsilon_{j+\frac{1}{2}}^{n}$, and the corresponding vectors $\boldsymbol{\sigma}_{h}^{n}$ and $\varepsilon_{h}^{n}$. For any discrete field $\mathbf{v}_{h}^{n}\left(\right.$ resp. $\mathbf{w}_{h}^{n}$ ) defined on $\Omega_{h, 0}$ (resp. $\Omega_{h, 1 / 2}$ ), we introduce the operator $\mathbf{D}_{h}$ (resp. $\mathbf{D}_{h}^{*}$ ) of second-order centered spatial derivative defined on $\Omega_{h, 1 / 2}\left(\right.$ resp. $\left.\Omega_{h, 0}\right)$ :

$$
\mathbf{D}_{h} \mathbf{v}_{h}^{n}=\left[\frac{v_{j+1}^{n}-v_{j}^{n}}{h}\right]_{j} \in \Omega_{h, 1 / 2}, \quad \mathbf{D}_{h}^{*} \mathbf{w}_{h}^{n}=\left[\frac{w_{j+\frac{1}{2}}^{n}-w_{j-\frac{1}{2}}^{n}}{h}\right]_{j} \in \Omega_{h, 0} .
$$

Finally, the full-discretized explicit problem writes:

$$
\begin{aligned}
\boldsymbol{\sigma}_{h k}^{n+1} & =H_{0 k} \mathbf{D}_{h} \mathbf{u}_{h}^{n+1}+p_{k} \boldsymbol{\sigma}_{h k}^{n}, \quad k=\left\{1, \ldots, N_{f}\right\}, \\
\boldsymbol{\sigma}_{h}^{n+1} & =H_{0} \mathbf{D}_{h} \mathbf{u}_{h}^{n+1}+\sum_{k=1}^{N_{f}} \boldsymbol{\sigma}_{h k}^{n+1}, \\
\rho \frac{\mathbf{u}_{h}^{n+1}-2 \mathbf{u}_{h}^{n}+\mathbf{u}_{h}^{n-1}}{\Delta t^{2}} & =\mathbf{D}_{h}^{*} \boldsymbol{\sigma}_{h}^{n}+\mathbf{f}_{h}^{n} .
\end{aligned}
$$

\subsection{Discrete energy and stability analysis}

An interesting way to study the behavior of a numerical time-integration scheme is to investigate its ability to preserve a discrete energy across the time 
steps of the simulation [6]. Let us note that such a study has been performed in [18] for a discretized system with a generalized Zener model. The same approach has been applied to the present scheme where the material law is represented by a digital filter. For the sake of clarity, only the results of the energetic analysis and the subsequent stability analysis will be exposed in the current section and we refer to the AppendixA for the corresponding developments. In particular, notations $\|\cdot\|_{0},\|\cdot\|_{\frac{1}{2}}$ and $(\cdot, \cdot)_{0},(\cdot, \cdot)_{\frac{1}{2}}$ refer to particular norms and scalar products detailed in equations (A.2a) and (A.2b).

Studying scheme (25) enables us to express the following energy identity:

$$
\frac{E_{h}^{n+\frac{1}{2}}-E_{h}^{n-\frac{1}{2}}}{\Delta t}=P_{h}^{n}-D_{h}^{n},
$$

which relates the variation of the numerical energy $E_{h}^{n+\frac{1}{2}}$ between time steps $t^{n-\frac{1}{2}}$ and $t^{n+\frac{1}{2}}$ to the work of the external forces $P_{h}^{n}$ and the dissipation $D_{h}^{n}$ arising from one-pole filters at time step $t^{n}$. This identity makes use of internal variables $\mathbf{s}_{h k}^{n}$, which are related to the dissipative part of each one-pole filter:

$$
\mathbf{s}_{h k}^{n}=\boldsymbol{\sigma}_{h k}^{n}-\frac{H_{0 k}}{1-p_{k}} \mathbf{D}_{h} \mathbf{u}_{h}^{n}, \quad k=\left\{1, \ldots, N_{f}\right\} .
$$

In the previous energy identity $(26), E_{h}^{n+\frac{1}{2}}$ may be split in three distinct terms $E_{h k}^{n+\frac{1}{2}}, E_{h f}^{n+\frac{1}{2}}$ and $E_{h s}^{n+\frac{1}{2}}$ :

$$
E_{h}^{n+\frac{1}{2}}=E_{h k}^{n+\frac{1}{2}}+E_{h f}^{n+\frac{1}{2}}+E_{h s}^{n+\frac{1}{2}}
$$

with:

$$
\begin{aligned}
E_{h k}^{n+\frac{1}{2}}= & \frac{\rho}{2}\left\|\frac{\mathbf{u}_{h}^{n+1}-\mathbf{u}_{h}^{n}}{\Delta t}\right\|_{0}^{2}, \\
E_{h f}^{n+\frac{1}{2}}= & \frac{1}{2}\left(H_{0}+\sum_{k=1}^{N_{f}} \frac{H_{0 k}}{1-p_{k}}\right)\left(\mathbf{D}_{h} \mathbf{u}_{h}^{n+1}, \mathbf{D}_{h} \mathbf{u}_{h}^{n}\right)_{\frac{1}{2}} \\
& -\sum_{k=1}^{N_{f}} \frac{1-p_{k}^{2}}{8 H_{0 k} p_{k}}\left(\left\|\mathbf{s}_{h k}^{n+1}\right\|_{\frac{1}{2}}^{2}+\left\|\mathbf{s}_{h k}^{n}\right\|_{\frac{1}{2}}^{2}\right), \\
E_{h s}^{n+\frac{1}{2}}= & -\frac{\Delta t^{2}}{4} \sum_{k=1}^{N_{f}}\left(\frac{\mathbf{s}_{h k}^{n+1}-\mathbf{s}_{h k}^{n}}{\Delta t}, \mathbf{D}_{h}\left(\frac{\mathbf{u}_{h}^{n+1}-\mathbf{u}_{h}^{n}}{\Delta t}\right)\right)_{\frac{1}{2}} .
\end{aligned}
$$

The first term $E_{h k}^{n+\frac{1}{2}}$ corresponds to the discrete kinetic energy of the system, while the second term $E_{h f}^{n+\frac{1}{2}}$ is related to the discrete internal energy stored 
in the digital filter. Finally, the last term $E_{h s}^{n+\frac{1}{2}}$ comes from the finite difference approximation. In the right-hand side of Eq. (26), the dissipation $D_{h}^{n}$, which involves mean values of $\mathbf{s}_{h k}$ between time steps, is simply the sum of the dissipation terms associated to each one-pole filter:

$$
D_{h}^{n}=-\sum_{k=1}^{N_{f}} \frac{\left(1-p_{k}\right)^{2}}{2 \Delta t H_{0 k} p}\left[\left\|\frac{\mathbf{s}_{h k}^{n+1}+\mathbf{s}_{h k}^{n}}{2}\right\|_{\frac{1}{2}}^{2}+\left\|\frac{\mathbf{s}_{h k}^{n}+\mathbf{s}_{h k}^{n-1}}{2}\right\|_{\frac{1}{2}}^{2}\right] .
$$

Finally, the work of the external forces is expressed as:

$$
P_{h}^{n}=\left(\mathbf{f}_{h}^{n}, \frac{\mathbf{u}_{h}^{n+1}-\mathbf{u}_{h}^{n-1}}{2 \Delta t}\right)_{0} .
$$

On the basis of the previous energetic identity, we study the conditions which ensure that the discrete energy $E_{h}^{n+\frac{1}{2}}$ and the dissipation term $D_{h}^{n}$ remains positive for each time step. Assuming the necessary condition (6) on the poles $p_{k}$ enabling causality and stability of the filter is fulfilled, this leads to additional sufficient conditions involving filter coefficients and filter response $H(z)$ at a specific point:

$$
\begin{aligned}
H(1) & \geq 0, \\
H_{0 k} p_{k} & \leq 0, \quad k=\left\{1, \ldots, N_{f}\right\} ;
\end{aligned}
$$

as well as discretization parameters:

$$
\Delta t \leq h \sqrt{\frac{\rho}{H(-1)}} .
$$

It is worth noting that the filter representing a material law has to be synthesized prior to the simulation process at a selected sampling frequency $f_{s}$, which therefore fixes the time step $\Delta t$ used for the simulation: $\Delta t=f_{s}^{-1}$. Consequently, it is more interesting to express previous stability condition in terms of the sampling frequency and continuous frequency response of the filter:

$$
\begin{aligned}
H^{c}(0) & \geq 0, \\
H_{0 k} p_{k} & \leq 0, \quad k=\left\{1, \ldots, N_{f}\right\}, \\
h & \geq h_{\min }=\frac{1}{f_{s}} \sqrt{\frac{H^{c}\left(\pi f_{s}\right)}{\rho}} .
\end{aligned}
$$

So far, it is interesting to note that the two first conditions (30a) and (30b) 255 are exactly the same as (16) and (18) introduced in Section 2.4, which both 
ensure the positivity of the static modulus and the dissipative behavior of each one-pole sub-filter involved in the discrete behavior law. At last, the third condition (30c) is a classic CFL stability condition on the space discretization parameter $h$ that involves the response of filter $H^{c}$ at the Nyquist frequency $\omega=\pi f_{s}$ or $f=f_{s} / 2$, which is the maximum valid frequency associated to the sampling rate $f_{s}$. Indeed, the ratio $\sqrt{\frac{H^{c}\left(\pi f_{s}\right)}{\rho}}$ may be interpreted as the maximum speed of longitudinal waves traveling into the medium.

\subsection{Dispersion and dissipation analysis}

Dispersion and dissipation properties are essential features of numerical schemes in the simulation of wave propagation phenomena. Unfortunately, although the literature contains a large numbers of works for conservative systems, there seems to be only few works devoted to the study of dissipative systems, among which the work by Robertsson et al. [29].

A first step to investigate the dispersion and dissipation properties of our numerical scheme is to determine the associated dispersion relation. To do so, we follow a classical procedure and consider a single wave solution of the form:

$$
\mathbf{u}_{h}^{n}=\left[w^{n} \exp \left(i j k_{h}^{*} h\right)\right]_{j}, \quad(\text { with } i=\sqrt{-1}),
$$

where $k_{h}^{*}$ is the complex wavenumber of the discrete problem accounting for both dispersion and dissipation phenomena. We also extend the definition (3) of the Z-transform to the fields defined on $\Omega_{h, 0}$ and $\Omega_{h, \frac{1}{2}}$ as:

$$
\mathrm{TZ}:\left\{\mathbf{v}_{h}^{n}\right\}_{n}=\left\{\left[v_{j}^{n}\right]_{j}\right\}_{n} \longmapsto \check{\mathbf{v}}_{h}(z)=\left[\sum_{n=-\infty}^{\infty} \mathbf{v}_{h}^{n} z^{-n}\right]_{j},
$$

such that the fundamental property $\mathrm{TZ}\left(\mathbf{v}_{h}^{n+1}\right)=z \mathrm{TZ}\left(\mathbf{v}_{h}^{n}\right)$ holds.

Then, applying the Z-tranform to Equation (25), where the external forces are taken equal to zero, gives:

$$
\begin{aligned}
\check{\boldsymbol{\sigma}}_{h k}(z)\left(1-p_{k} z^{-1}\right) & =H_{0 k} \mathrm{TZ}\left(\mathbf{D}_{h} \mathbf{u}_{h}^{n}\right), \quad k=\left\{1, \ldots, N_{f}\right\}, \\
\check{\boldsymbol{\sigma}}_{h}(z) & =H_{0} \mathrm{TZ}\left(\mathbf{D}_{h} \mathbf{u}_{h}^{n}\right)+\sum_{k=1}^{N_{f}} \check{\boldsymbol{\sigma}}_{h k}(z), \\
\rho \frac{z-2+z^{-1}}{\Delta t^{2}} \check{\mathbf{u}}_{h}(z) & =\mathrm{TZ}\left(\mathbf{D}_{h}^{*} \boldsymbol{\sigma}_{h}^{n}\right) .
\end{aligned}
$$


From the linearity of TZ, one has $\operatorname{TZ}\left(\mathbf{D}_{h} \mathbf{v}_{h}^{n}\right)=\mathbf{D}_{h} \check{\mathbf{v}}_{h}(z)$ and the same applies to $\mathbf{D}_{h}^{*}$, such that Equation (33) may be rewritten involving discrete transfer function $H(z)$ by substituting (33a) into (33b):

$$
\rho \check{\delta}_{t} \cdot(z) \check{\mathbf{u}}_{h}(z)=H(z) \mathbf{D}_{h}^{*} \mathbf{D}_{h} \check{\mathbf{u}}_{h}(z),
$$

where $\check{\delta}_{t} .(z)$ denotes the $\mathrm{Z}$ transform of the leap-frog scheme (25b):

$$
\check{\delta}_{t} \cdot(z)=\frac{z-2+z^{-1}}{\Delta t^{2}} .
$$

Next, using the discrete-time-space ansatz (31) leads to:

$$
\mathbf{D}_{h}^{*} \mathbf{D}_{h} \check{\mathbf{u}}_{h}(z)=\frac{\exp \left(i k_{h}^{*} h\right)-2+\exp \left(-i k_{h}^{*} h\right)}{h^{2}} \check{\mathbf{u}}_{h}(z)=-\frac{4}{h^{2}} \sin ^{2}\left(\frac{k_{h}^{*} h}{2}\right) \check{\mathbf{u}}_{h}(z),
$$

so that Equation (34) becomes:

$$
\rho \check{\delta}_{t} \cdot(z)=-\frac{4}{h^{2}} H(z) \sin ^{2}\left(\frac{k_{h}^{*} h}{2}\right) .
$$

Finally, the dispersion relation may be obtained by evaluating relation (36) on the unit circle, that is substituting $z$ by $\exp \left(i \omega f_{s}^{-1}\right)$ and observing that:

$$
\check{\delta}_{t \cdot}\left(\exp \left(i \omega f_{s}^{-1}\right)\right)=-\frac{4}{\Delta t^{2}} \sin ^{2}\left(\frac{\omega \Delta t}{2}\right) .
$$

As a result, we obtain the following dispersion relation:

$$
k_{h}^{*}(\omega)=k_{h r}(\omega)+i k_{h i}(\omega)= \pm \frac{2}{h} \arcsin \left[\frac{h}{\Delta t} \sqrt{\frac{\rho}{H^{c}(\omega)}} \sin \left(\frac{\omega \Delta t}{2}\right)\right] .
$$

From the real part $k_{h r}$ and imaginary part $k_{h i}$ of the discrete wave number, we also define the speed $c_{h}$ and damping coefficient $\alpha_{h}$ associated with the discrete problem (25). To do so, we consider a continuous fictive solution $u$ which may be written as:

$$
\begin{aligned}
u(x, t) & =u_{0} \exp \left(i k_{h}^{*}(\omega) x-\omega t\right) \\
& =u_{0} \exp \left(-k_{h i}(\omega) x\right) \exp \left(i k_{h r}(\omega) x-\omega t\right) \\
& =u_{0} \exp \left(-\alpha_{h}(\omega) t\right) \exp \left(i k_{h r}(\omega)\left(x-c_{h}(\omega) t\right),\right.
\end{aligned}
$$

with $c_{h}$ and $\alpha_{h}$ defined as:

$$
c_{h}(\omega)=\frac{\omega}{k_{h r}(\omega)}, \quad \alpha_{h}(\omega)=c_{h}(\omega) k_{h i}(\omega)=\omega \frac{k_{h i}(\omega)}{k_{h r}(\omega)} .
$$




\section{Numerical simulations}

Let us now illustrate on a simple example the whole approach, including both filter synthesis and simulation steps. To do so, we consider the model problem of Section 2.1 with a beam of length $L=1 \mathrm{~m}$ and density $\rho=1149 \mathrm{~kg} / \mathrm{m}^{3}$, constituted of the same material as the nylon bar specimen studied by Collet et al. [22] and excited by an impact on its right end. In order to build the discrete-time behavior, we consider the experimental data on complex modulus obtained in [22] and represented (with black dots) on Figure 6.

In the following, we wish to simulate the response of the beam on the frequency range $[20 \mathrm{~Hz}, 20 \mathrm{kHz}]$ corresponding to audible signals, while ensuring given error criteria on dispersion and dissipation. Concerning sound perception, the most restrictive criterion has to be set on the dispersion error which will be limited to $1 \%$ as it corresponds to the order of magnitude of pitch sensitivity perceived by the human ear. The criterion on the dissipation error, which is related to the temporal decay of each harmonics, is less critical and will be 285 set accordingly to $5 \%$.

Next, in the present discrete-time behavior framework, the whole simulation process process involves: (1) finding the best sampling frequency $f_{s},(2)$ identifying material law and (3) performing numerical simulation. However, choosing the best sampling frequency $f_{s}$ from an error criterion on dispersion and dissipation of the numerical scheme is not an easy task since the validation of such criterion depends on the continuous response of the synthesized filter, as shown in Section 4.3, which itself requires $f_{s}$ to be fixed prior to the optimization process, as shown in Section 3. Consequently, in order to minimize overall computational load, we suggest to set $f_{s}$ to its lowest possible value through an iterative procedure that involves many filter synthesis followed by validation of dispersion and dissipation error criteria.

\subsection{Filter synthesis}

In the subsequent developments, for the sake of simplicity, we choose to fix the sampling frequency $f_{s}$ a priori to $180 \mathrm{kHz}$, which is 9 times higher than the maximum frequency of the considered frequency range. Then, filter $\mathcal{H}$ is synthesized through constrained optimization problem (20), using GNU Octave 
sqp function in a same way as what has been done in Section 3.2. At the end of the process, one obtains a filter made of $N_{f}=4$ one-pole sub-filter whose coefficients are detailed in Table 2 and whose frequency-domain response is plotted in Figure 6. It must be emphasized that the synthesized filter can only represent the correct material behavior in the frequency range covered by the experimental values, i.e. $500 \mathrm{~Hz}$ to $8 \mathrm{kHz}$ approximately. Outside this frequency range, the filter yields a physically possible behavior in terms of causality and verification of thermodynamics laws, but not necessarily the "correct" behavior for such materials. In the following, we set the frequency range of interest $\left[f_{\min }, f_{\max }\right]$ (introduced in Section 3.2) of the synthesis algorithm to [100 Hz, $20 \mathrm{kHz}]$. This frequency range thus goes beyond the frequency range covered by the experimental values of Collet et al. [22].
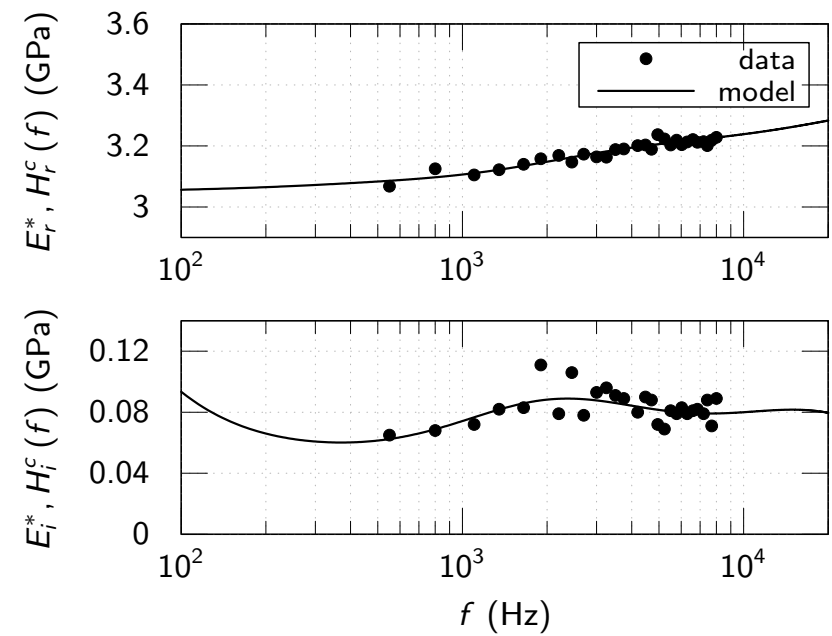

Figure 6: Real (top) and imaginary (bottom) parts of experimental data (solid circles) related to the nylon bar specimen of [22] and response $H^{c}$ of the synthesized digital filter (solid curves) with pure gain and $N_{f}=4$ one-pole sub-filters.

\subsection{Choice of excitation function}

In order to highlight the influence of dispersion and dissipation due to the numerical scheme during the time-domain simulation, it is desirable to use a broadband excitation with small low frequency components. Thus, we choose 


\begin{tabular}{rl}
\hline$H_{0}, H_{0, k}$ & $p_{k}$ \\
\hline $3.4299 \cdot 10^{9}$ & \\
$-2.8670 \cdot 10^{5}$ & $9.8987 \cdot 10^{-1}$ \\
$-2.7138 \cdot 10^{5}$ & $9.9991 \cdot 10^{-1}$ \\
$-1.0050 \cdot 10^{7}$ & $9.2889 \cdot 10^{-1}$ \\
$-1.0941 \cdot 10^{8}$ & $4.6631 \cdot 10^{-1}$ \\
\hline
\end{tabular}

Table 2: Coefficients of synthesized filter $\mathcal{H}$ related to Nylon for $N_{f}=4$.

to model the impact time function by a Ricker wavelet given by:

$$
F(t)=F_{0}\left(1-2 \pi^{2} f_{0}^{2}\left(t-t_{0}\right)^{2}\right) \exp \left(-\pi^{2} f_{0}^{2}\left(t-t_{0}\right)^{2}\right)
$$

where $f_{0}$ is the maximum of the frequency spectrum, fixed to $10 \mathrm{kHz}$, and $F_{0}=$ $1.0 \cdot 10^{11} \mathrm{~N} / \mathrm{m}^{2}$. Figure 7 shows the time-domain shape and the power spectral density of such an excitation. Let us note that all power spectral densities
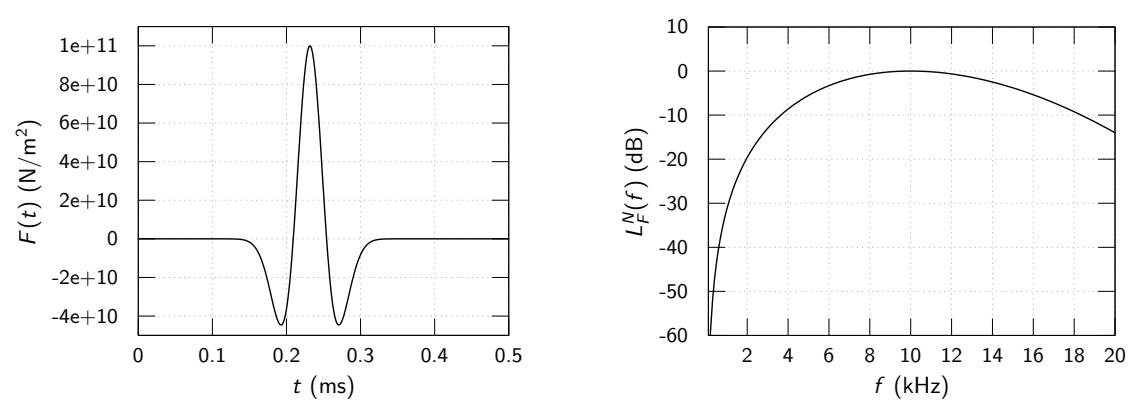

Figure 7: Time-domain evolution (left) and normalized power spectral density (right) of Ricker wavelet excitation $F(t)$ with $f_{0}=10 \mathrm{kHz}$.

presented here are given in terms of normalized power level in $\mathrm{dB}$, which writes for a given signal $\hat{v}(f)$ as:

$$
L_{V}^{N}=20 \log _{10}\left(\frac{\hat{v}(f)}{\max _{f} \hat{v}(f)}\right) .
$$

\subsection{Time-domain simulation}

Once the filter has been synthesized, numerical simulation of the resulting discrete problem (25) is performed keeping the same time discretization corresponding to the sampling frequency $f_{s}$. Then, the CFL condition (30c) associated with the explicit scheme (25) gives the minimum value $h_{\min }$ of the spatial 
discretization parameter $h$ in order to guarantee the stability of the numerical scheme. Next, mesh size is chosen as the minimum value $h \geq h_{\min }$ which remains compatible with the uniform spatial discretization of the beam, which yields (denoting $E(Y)$ the integer part of $Y$ ):

$$
h=\frac{L}{N_{h}} \quad \text { with } \quad N_{h}=E\left(\frac{L}{h_{\min }}\right)+1 .
$$

Furthermore, it is interesting to note from (30c) the proportionality relation between $h_{\min }$ and $\sqrt{H^{c}\left(\pi f_{s}\right)}$, which suggests to keep this last value as small as possible during the optimization process in order to lower the spatial discretization step. This may be particularly relevant when working in a frequency range $\left[f_{\min }, f_{\max }\right]$ that is far from $f_{s} / 2$, where the optimization process may leads to a significant increase of $H^{c}$ between $f_{\max }$ and $f_{s} / 2$ without noticeable improvement of the material response on the frequency range of interest. This last remark may be taken into account by directly incorporating an additional constraint $H^{c}\left(\pi f_{s}\right) \leq H_{\max }^{c}$ in the optimization process (20).

In order to study the influence of $h$ on the properties of the numerical scheme, let us introduce the following CFL number as $\mathrm{CFL}=\frac{h}{h_{\min }}$, such that $\mathrm{CFL}=1$ in the ideal case corresponding to $h=h_{\min }$. As for the present problem, the minimum possible spatial size is $h=9.52 \mathrm{~mm}$ which corresponds to a CFL number of 1.004. The time evolution of beam displacement $u$ at observation point $x_{\mathrm{obs}}=0.47 \mathrm{~L}$ is plotted on Figure 8 . Figure 9 plots both the associated power spectral density $L_{U}^{N}$ and phase $\varphi_{U}$ for the two CFL numbers and compares it to a reference modal solution detailed in AppendixB. These two figures show that the numerical solution agrees very well with the modal one over the whole frequency band for the best CFL value of 1.004, whereas one may clearly notice some discrepancies increasing with frequency for a CFL value of 1.110 .

Now, in order to illustrate the performance of the scheme relative to dispersion and dissipation, we introduce the relative errors $e_{r}^{k}$ and $e_{i}^{k}$ on numerical dispersion and dissipation by:

$$
e_{r}^{k}(\omega)=\frac{\left|k_{r}(\omega)-k_{h r}(\omega)\right|}{\left|k_{r}(\omega)\right|}, \quad e_{i}^{k}(\omega)=\frac{\left|k_{i}(\omega)-k_{h i}(\omega)\right|}{\left|k_{i}(\omega)\right|},
$$

where $k_{h r}$ and $k_{h i}$ are defined from dispersion analysis of Section 4.3 and $k_{r}$ and $k_{i}$ are related to the complex number of the reference problem. Figure 10 shows relatives errors $e_{r}^{k}$ and $e_{i}^{k}$ on the real and imaginary part of the wavenumber for 

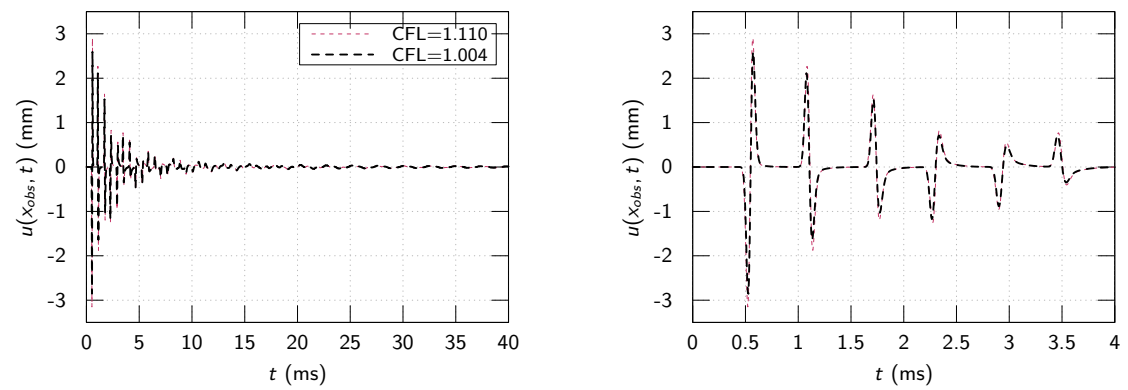

Figure 8: Time-domain response $u\left(x_{\mathrm{obs}}, t\right)$ corresponding to CFL $=1.004$ (black thick dashed) and $\mathrm{CFL}=1.110$ (magenta thin dashed) up to $40 \mathrm{~ms}$ (left) and up to $4 \mathrm{~ms}$ (right).
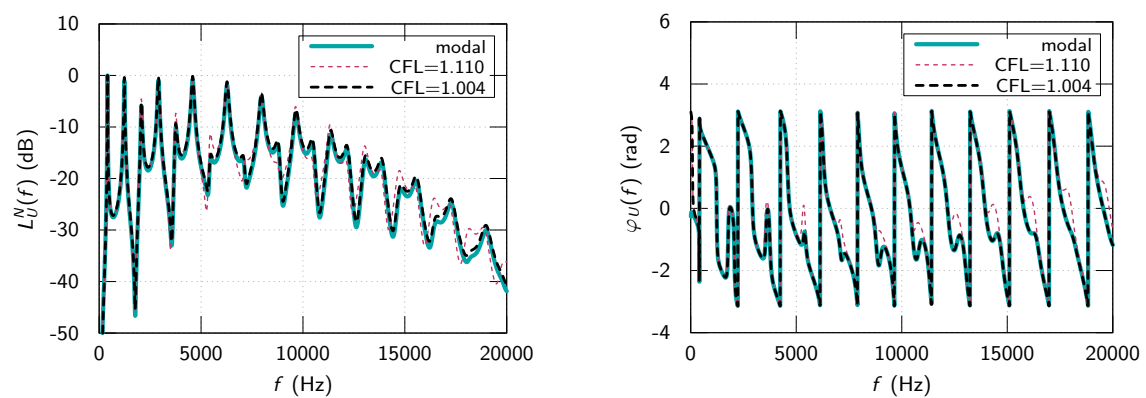

Figure 9: Power spectral density $L_{U}^{N}$ in log level (left) and phase response $\varphi_{U}$ (right) corresponding to $\mathrm{CFL}=1.004$ (black thick dashed), $\mathrm{CFL}=1.110$ (magenta thin dashed) and reference solution (cyan thick solid).

the ideal case corresponding to $\mathrm{CFL}=1$ (solid curve) and for the lower admissible spatial discretization corresponding to $\mathrm{CFL}=1.004$ (dashed curve). We notice that the error $e_{r}^{k}$, which may be linked to the speed of waves propagating into the medium, remains lower than $1 \%$ over the whole frequency range of interest. However, comparing it with the ideal curve shows an important variation of $e_{r}^{k}$ close to $f_{s} / 2$ despite a very low increase of the CFL coefficient. As for the imaginary part of the wavenumber, it is noticeable that the relative error is an order of magnitude greater. However, we notice that the increase of CFL number has less influence here, the two corresponding curves being very close. Hence, the dissipation error seems to come essentially from the numerical scheme itself and little from the choice of discretization parameters, which suggest to use fourth order schemes in the time discretization of Equation (1b) and in the definition of spatial operators (24) in order to reduce it. Here, optimal choice 


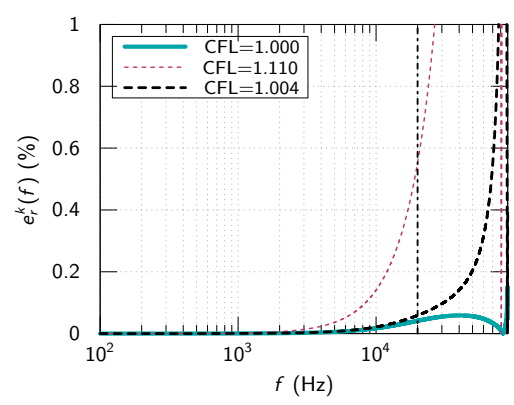

(a) Dispersion error $e_{r}^{k}(\omega)$

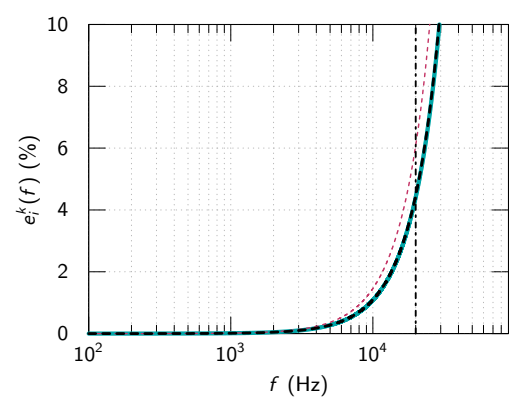

(b) Dissipation error $e_{i}^{k}(\omega)$

Figure 10: Dispersion and dissipation properties corresponding to CFL=1 (cyan thick solid), $\mathrm{CFL}=1.004$ (black thick dashed) and $\mathrm{CFL}=1.110$ (magenta thin dashed) on the frequency range $\left[f_{\min }, f_{s} / 2\right]$, where the maximum frequency of interest $f_{\max }=20 \mathrm{kHz}$ is shown by dashed black line.

of $h$ corresponding to $\mathrm{CFL}=1.004$ leads to a relative error of $0.06 \%$ for $e_{r}^{k}$ and $4.46 \%$ for $e_{i}^{k}$ at $20 \mathrm{kHz}$, which validates a posteriori the initial choice of $f_{s}$ regarding error criterions introduced in the beginning of the section.

Starting from the dispersion relation, one can express the wavelength $\lambda$ as a function of the frequency $f$ for the continuous reference problem by:

$$
\lambda(f)=c(f) f^{-1}=\frac{\mathcal{R} e\left(H^{c}(f)\right)}{f \sqrt{\rho}} .
$$
for the dissipation, the increase of the relative error is also significant with $e^{k}$ reaching $6.06 \%$, which leads to an underestimation of damping on the higher frequency range. This last point is also noticeable with overruns located on the 
peaks of the time-domain responses plotted on Figure 8.

\section{Conclusion}

The work that has been presented in this paper introduces an original approach to provide well-posed and accurate description of damping phenomena for various materials in the context of time-domain numerical simulations. Its original feature is to build a discrete-time model which may be interpreted as a digital filter, thus allowing to use the common tools available in the field of digital filtering. The whole process including material identification and numerical simulation may be summarized by the following steps:

1. choose an initial sampling frequency $f_{s}$;

2. synthesize filter $\mathcal{H}$, representing discrete-time material law, from experimental data;

3. calculate minimum grid step $h_{\min }$ from $H^{c}\left(\pi f_{s}\right)$ using CFL relation (30c);

4. choose minimal grid step $h \geq h_{\min }$ compatible with geometric parameters;

5. compute dispersion and dissipation errors $e_{r}^{k}$ and $e_{i}^{k}$ over the frequency range and validate error criterion;

6. if previous validation fails, choose a refined sampling frequency $f_{s}^{\prime} \geq f_{s}$ and restart steps 2 to 5 up to the satisfaction of error criterion.

The filter is synthesized through an optimization process in order to have its transfer function in the frequency domain approximate a given set of complex elastic moduli at various frequencies. Moreover, adding some constraints on the filter coefficients during the optimization process allows one to obtain a resulting discrete-time model satisfying stability, causality and positivity of dissipation. Finally, its transposition into the time domain is straightforward and leads to a recursive discrete-time relation which is directly implementable into a numerical integration scheme and may be interpreted as a discrete-time constitutive law with internal variables. Furthermore, the choice of the subfilters constituting the filter previously synthesized, enable the whole numerical scheme resulting from the discrete-time constitutive law and the discretization of linear momentum equation to fulfill some discrete-time energetic identity. As a result, the whole modeling procedure may be interpreted as a two-way 
400 process which (1) carries identification of a continuous time-domain law from experimental results and (2) sets up the best time-discretization scheme in order to master the high-frequency discrepancies between continuous-time and discrete-time frequency response of the resulting model. Besides, one may evaluate errors on dispersion and dissipation of the numerical scheme and control

405 it through an optimal choice of both discretization and filter parameters having an impact on the high-frequency response of the discrete model.

Up to now, this approach has been developed on a one-dimensional problem with finite difference integration scheme in space and time. Future developments will deal with its integration into dynamic three dimensional problems with finite element discretization in space and finite difference time integration, and with extension to inhomogeneous and anisotropic materials. Also, the extension and theoretical analysis of the general form of the material filter with multiple and complex-conjugate poles will be done in future works.

\section{Acknowledgments}

The authors would like to thank the French « Direction Générale de l'Armement » (DGA) for supporting this work.

\section{References}

[1] S. Crandall, The role of damping in vibration theory, Journal of Sound and Vibration 11 (1) (1970) 3-18, doi:10.1016/S0022-460X(70)80105-5.

[2] L. Gaul, The Influence of Damping on Waves and Vibrations, Mechanical Systems and Signal Processing 13 (1) (1999) 1-30, doi: 10.1006/mssp.1997.0185.

[3] M. D. Rao, Recent applications of viscoelastic damping for noise control in automobiles and commercial airplanes, Journal of Sound and Vibration 262 (3) (2003) 457-474, doi:10.1016/S0022-460X(03)00106-8.

[4] T. X. Wu, D. J. Thompson, On the impact noise generation due to a wheel passing over rail joints, Journal of Sound and Vibration 267 (3) (2003) 485-496, doi:10.1016/S0022-460X(03)00709-0. 
[5] M. Vorländer, Auralization of spaces, Physics Today 62 (6) (2009) 35-40, doi:10.1063/1.3156831.

[6] S. Bilbao, Numerical Sound Synthesis, Wiley, 1 edn., ISBN 9780470510469, 2009.

[7] A. Chaigne, V. Doutaut, Numerical simulations of xylophones. I. Timedomain modeling of the vibrating bars, The Journal of the Acoustical Society of America 101 (1) (1997) 539-557, doi:10.1121/1.418117.

[8] E. Bécache, A. Chaigne, G. Derveaux, P. Joly, Numerical simulation of a guitar, Computers \& Structures 83 (2-3) (2005) 107-126, doi: 10.1016/j.compstruc.2004.04.018.

[9] J. Chabassier, A. Chaigne, P. Joly, Modeling and simulation of a grand piano., J. Acoust. Soc. Am. 134 (1) (2013) 648-665, doi:10.1121/1.4809649.

[10] S. McAdams, V. Roussarie, A. Chaigne, B. L. Giordano, The psychomechanics of simulated sound sources: material properties of impacted thin plates., J. Acoust. Soc. Am. 128 (3) (2010) 1401-1413, doi: 10.1121/1.3466867.

[11] J. Soovere, M. Drake, Aerospace structures technology damping design guide, Volume III - Damping material data, AFWAL-TR-84-3089, Air Force Wright Aeronautical Laboratories, 1985.

[12] T. Pritz, Frequency power law of material damping, Applied Acoustics 65 (11) (2004) 1027-1036, doi:10.1016/j.apacoust.2004.06.001.

[13] R. M. Christensen, Theory of Viscoelasticity, Civil, Mechanical and Other Engineering Series, Dover, ISBN 9780486428802, 2003.

[14] D. F. Golla, P. C. Hughes, Dynamics of Viscoelastic Structures-A TimeDomain, Finite Element Formulation, Journal of Applied Mechanics 52 (4) (1985) 897-906, doi:10.1115/1.3169166.

${ }_{455}$ [15] D. J. McTavish, P. C. Hughes, Modeling of Linear Viscoelastic Space Structures, Journal of Vibration and Acoustics 115 (1) (1993) 103-110, doi: 10.1115/1.2930302. 
[16] G. Lesieutre, E. Bianchini, Time Domain Modeling of Linear Viscoelasticity Using Anelastic Displacement Fields, Journal of Vibration and Acoustics 117 (1995) 424-430.

[17] A. Chaigne, C. Lambourg, Time-domain simulation of damped impacted plates. I. Theory and experiments, The Journal of the Acoustical Society of America 109 (4) (2001) 1422-1432, doi:10.1121/1.1354200.

[18] E. Bécache, A. Ezziani, P. Joly, A mixed finite element approach for viscoelastic wave propagation, Computational Geosciences 8 (3) (2005) 255299, doi:10.1007/s10596-005-3772-8.

[19] R. L. Bagley, P. J. Torvik, Fractional calculus - A different approach to the analysis of viscoelastically damped structures, AIAA Journal 21 (5) (1983) 741-748, doi:10.2514/3.8142.

470 [20] A. Lion, On the thermodynamics of fractional damping elements, Continuum Mechanics and Thermodynamics 9 (2) (1997) 83-96, doi: $10.1007 / \mathrm{s} 001610050057$.

[21] J.-F. Deü, D. Matignon, Simulation of fractionally damped mechanical systems by means of a Newmark-diffusive scheme, Computers \& Mathematics with Applications 59 (5) (2010) 1745-1753, doi: 10.1016/j.camwa.2009.08.067.

[22] P. Collet, G. Gary, B. Lundberg, Noise-Corrected Estimation of Complex Modulus in Accord With Causality and Thermodynamics: Application to an Impact Test, Journal of Applied Mechanics 80 (1) (2013) 1018-1024, doi:10.1115/1.4007081.

[23] J. O. Smith, Introduction to Digital Filters with Audio Applications, Music signal processing series, W3K Publishing, ISBN 9780974560717, 2007.

[24] A. V. Oppenheim, R. W. Schafer, Discrete-Time Signal Processing, Prentice Hall, 3 edn., ISBN 9780132146357, 2009.

${ }_{485}$ [25] F. Renaud, J.-L. Dion, G. Chevallier, I. Tawfiq, R. Lemaire, A new identification method of viscoelastic behavior: Application to the generalized 
Maxwell model, Mechanical Systems and Signal Processing 25 (3) (2011) 991-1010, doi:10.1016/j.ymssp.2010.09.002.

[26] J. Bonnans, J. Gilbert, C. Lemaréchal, C. Sagastizábal, Numerical Optimization, Theoretical and Practical Aspects, Springer-Verlag, second edn., ISBN $9783540354475,2006$.

[27] M. Mossberg, L. Hillström, T. Söderström, Non-parametric identification of viscoelastic materials from wave propagation experiments, Automatica 37 (4) (2001) 511-521, doi:10.1016/S0005-1098(00)00188-6.

${ }_{495}$ [28] R. Pintelon, P. Guillaume, S. Vanlanduit, K. De Belder, Y. Rolain, Identification of Young's modulus from broadband modal analysis experiments, Mechanical Systems and Signal Processing 18 (4) (2004) 699-726, doi: 10.1016/S0888-3270(03)00045-1.

[29] J. Robertsson, J. Blanch, W. Symes, Viscoelastic finite-difference modeling, Geophysics 59 (9) (1994) 1444-1456, doi:10.1190/1.1443701.

\section{AppendixA. Energetic analysis and stability of integration scheme}

In order to perform the energetic analysis of scheme (25), we first start by introducing the Hilbert spaces $L^{2}\left(\Omega_{h, 0}\right)$ et $L^{2}\left(\Omega_{h, 1 / 2}\right)$ defined as:

$$
\begin{aligned}
L^{2}\left(\Omega_{h, 0}\right) & =\left\{\mathbf{v}_{h}=\left[v_{j}\right]_{j=\left\{0, \ldots, N_{h}\right\}} \in \Omega_{h, 0}, \sum_{j=0}^{N_{h}}\left|v_{j}\right|^{2}<+\infty\right\}, \\
L^{2}\left(\Omega_{h, 1 / 2}\right) & =\left\{\mathbf{v}_{h}=\left[v_{j+\frac{1}{2}}\right]_{j=\left\{0, \ldots, N_{h}-1\right\}} \in \Omega_{h, 1 / 2}, \sum_{j=0}^{N_{h}-1}\left|v_{j+\frac{1}{2}}\right|^{2}<+\infty\right\} ;
\end{aligned}
$$


together with the following associated discrete norms and scalar products:

$$
\begin{aligned}
&\left\|\mathbf{v}_{h}\right\|_{0}=\left[\sum_{j=0}^{N_{x}} h\left|v_{j}\right|^{2}\right]^{\frac{1}{2}}, \quad\left(\mathbf{v}_{h}, \mathbf{w}_{h}\right)_{0}=\sum_{j=0}^{N_{x}} h v_{j} w_{j}, \\
& \forall\left(\mathbf{v}_{h}, \mathbf{w}_{h}\right) \in L^{2}\left(\Omega_{h, 0}\right)^{2}, \\
&\left\|\mathbf{v}_{h}\right\|_{\frac{1}{2}}=\left[\sum_{j=0}^{N_{x}-1} h\left|v_{j+\frac{1}{2}}\right|^{2}\right]^{\frac{1}{2}}, \quad\left(\mathbf{v}_{h}, \mathbf{w}_{h}\right)_{\frac{1}{2}}=\sum_{j=0}^{N_{x}-1} h v_{j+\frac{1}{2}} w_{j+\frac{1}{2}}, \\
& \forall\left(\mathbf{v}_{h}, \mathbf{w}_{h}\right) \in L^{2}\left(\Omega_{h, 1 / 2}\right)^{2} .
\end{aligned}
$$

Let us note that $\mathbf{D}_{h}^{*}$ and $\mathbf{D}_{h}$ are skew-self-adjoint operators, such that:

$$
\left(\mathbf{v}_{h}, \mathbf{D}_{h}^{*} \mathbf{w}_{h}\right)_{0}=-\left(\mathbf{D}_{h} \mathbf{v}_{h}, \mathbf{w}_{h}\right)_{\frac{1}{2}}, \quad \forall\left(\mathbf{v}_{h}, \mathbf{w}_{h}\right) \in L^{2}\left(\Omega_{h, 0}\right) \times L^{2}\left(\Omega_{h, 1 / 2}\right) .
$$

For the sake of simplicity, we perform the stability analysis on numerical schemes with increasing complexity, starting with a system with one pure gain filter, followed by a system with one-pole filter, before extending it to system (25) of multiple one-pole filters. Finally, this last energetic result allows us to derive the stability condition of scheme (25).

\section{AppendixA.1. System with pure gain filter}

We start by performing the energetic analysis of a simplified discrete problem where the material behavior is only constituted by a pure gain filter $(H(z)=$ $H_{0}$ ), corresponding to a purely elastic case. Equation (25a) becomes $\boldsymbol{\sigma}_{h}^{n}=$ $H_{0} \mathbf{D}_{h} \mathbf{u}_{h}^{n}$ and the discrete problem associated with this lossless system writes:

$$
\rho \frac{\mathbf{u}_{h}^{n+1}-2 \mathbf{u}_{h}^{n}+\mathbf{u}_{h}^{n-1}}{\Delta t^{2}}=H_{0} \mathbf{D}_{h}^{*} \mathbf{D}_{h} \mathbf{u}_{h}^{n}+\mathbf{f}_{h}^{n} .
$$

Next, the energetic identity is achieved by multiplying (A.3) by $\left(\mathbf{u}_{h}^{n+1}-\mathbf{u}_{h}^{n-1}\right) / 2 \Delta t$, which is the second order centered approximation of $\partial u / \partial t$ at step $t^{n}$, and integrating it on the whole domain. Using the norms and scalar products previously introduced yields:

$$
\begin{aligned}
\frac{\rho}{2 \Delta t}\left[\left\|\frac{\mathbf{u}_{h}^{n+1}-\mathbf{u}_{h}^{n}}{\Delta t}\right\|_{0}^{2}-\left\|\frac{\mathbf{u}_{h}^{n}-\mathbf{u}_{h}^{n-1}}{\Delta t}\right\|_{0}^{2}\right] & +H_{0}\left(\mathbf{D}_{h} \mathbf{u}_{h}^{n}, \mathbf{D}_{h}\left(\frac{\mathbf{u}_{h}^{n+1}-\mathbf{u}_{h}^{n-1}}{2 \Delta t}\right)\right)_{\frac{1}{2}} \\
& =\left(\mathbf{f}_{h}^{n}, \frac{\mathbf{u}_{h}^{n+1}-\mathbf{u}_{h}^{n-1}}{2 \Delta t}\right)_{0} .
\end{aligned}
$$


Then, one obtains the following energetic identity:

$$
\frac{E_{h}^{n+\frac{1}{2}}-E_{h}^{n-\frac{1}{2}}}{\Delta t}=\left(\mathbf{f}_{h}^{n}, \frac{\mathbf{u}_{h}^{n+1}-\mathbf{u}_{h}^{n-1}}{2 \Delta t}\right)_{0},
$$

where $E_{h}^{n+\frac{1}{2}}$ stands for the discrete energy of the system at time step $t^{n+\frac{1}{2}}$, which is defined as:

$$
E_{h}^{n+\frac{1}{2}}=\frac{\rho}{2}\left\|\frac{\mathbf{u}_{h}^{n+1}-\mathbf{u}_{h}^{n}}{\Delta t}\right\|_{0}^{2}+\frac{H_{0}}{2}\left(\mathbf{D}_{h} \mathbf{u}_{h}^{n+1}, \mathbf{D}_{h} \mathbf{u}_{h}^{n}\right) .
$$

From a mechanical point of view, $E_{h}^{n+\frac{1}{2}}$ stands for the classical discrete energy corresponding to a purely elastic behavior [18], and identity (A.5) simply expresses that its variation between time steps $t^{n-\frac{1}{2}}$ and $t^{n+\frac{1}{2}}$ is equal to the power of external forces $\mathbf{f}_{h}^{n}$ at time step $t^{n}$.

AppendixA.2. System with one-pole filter

Now, let us consider the case of a simplified discrete problem where the material behavior is only constituted by a one-pole filter:

$$
\begin{aligned}
\boldsymbol{\sigma}_{h}^{n+1} & =H_{0} \mathbf{D}_{h} \mathbf{u}_{h}^{n+1}+p \boldsymbol{\sigma}_{h}^{n}, \\
\rho \frac{\mathbf{u}_{h}^{n+1}-2 \mathbf{u}_{h}^{n}+\mathbf{u}_{h}^{n-1}}{\Delta t^{2}} & =\mathbf{D}_{h}^{*} \boldsymbol{\sigma}_{h}^{n}+\mathbf{f}_{h}^{n} .
\end{aligned}
$$

First of all, we introduce the internal variable $\mathbf{s}_{h}^{n}$ as:

$$
\mathbf{s}_{h}^{n}=\boldsymbol{\sigma}_{h}^{n}-\frac{H_{0}}{1-p} \mathbf{D}_{h} \mathbf{u}_{h}^{n},
$$

in order to rewrite System (A.7) in terms of $\mathbf{s}_{h}^{n}$ :

$$
\begin{aligned}
\frac{1-p}{2}\left(\mathbf{s}_{h}^{n+1}+\mathbf{s}_{h}^{n}\right)+\frac{1+p}{2}\left(\mathbf{s}_{h}^{n+1}-\mathbf{s}_{h}^{n}\right) & =-\frac{H_{0} p}{1-p} \mathbf{D}_{h}\left(\mathbf{u}_{h}^{n+1}-\mathbf{u}_{h}^{n}\right) . \\
\rho \frac{\mathbf{u}_{h}^{n+1}-2 \mathbf{u}_{h}^{n}+\mathbf{u}_{h}^{n-1}}{\Delta t^{2}} & =\mathbf{D}_{h}^{*} \mathbf{s}_{h}^{n}+\frac{H_{0}}{1-p} \mathbf{D}_{h}^{*} \mathbf{D}_{h} \mathbf{u}_{h}^{n}+\mathbf{f}_{h}^{n} .
\end{aligned}
$$

Next, one multiplies (A.9a) by $\frac{\mathbf{s}_{h}^{n+1}+\mathbf{s}_{h}^{n}}{2}$ and integrates over the whole domain, while processing equilibrium Equation (A.9b) in a similar way as for the lossless 
System (A.4), which yields:

$$
\begin{aligned}
& \frac{1-p}{2}\left(\mathbf{s}_{h}^{n+1}+\mathbf{s}_{h}^{n}, \frac{\mathbf{s}_{h}^{n+1}+\mathbf{s}_{h}^{n}}{2}\right)_{\frac{1}{2}}+\frac{1+p}{2}\left(\mathbf{s}_{h}^{n+1}-\mathbf{s}_{h}^{n}, \frac{\mathbf{s}_{h}^{n+1}+\mathbf{s}_{h}^{n}}{2}\right)_{\frac{1}{2}} \\
& =-\frac{H_{0} p}{1-p}\left(\mathbf{D}_{h}\left(\mathbf{u}_{h}^{n+1}-\mathbf{u}_{h}^{n}\right), \frac{\mathbf{s}_{h}^{n+1}+\mathbf{s}_{h}^{n}}{2}\right)_{\frac{1}{2}} \\
& \frac{\rho}{2 \Delta t}\left[\left\|\frac{\mathbf{u}_{h}^{n+1}-\mathbf{u}_{h}^{n}}{\Delta t}\right\|_{0}^{2}-\left\|\frac{\mathbf{u}_{h}^{n}-\mathbf{u}_{h}^{n-1}}{\Delta t}\right\|_{0}^{2}\right] \\
& +\left(\mathbf{s}_{h}^{n}, \mathbf{D}_{h}\left(\frac{\mathbf{u}_{h}^{n+1}-\mathbf{u}_{h}^{n-1}}{2 \Delta t}\right)\right)_{\frac{1}{2}}+\frac{H_{0}}{1-p}\left(\mathbf{D}_{h} \mathbf{u}_{h}^{n}, \mathbf{D}_{h}\left(\frac{\mathbf{u}_{h}^{n+1}-\mathbf{u}_{h}^{n-1}}{2 \Delta t}\right)\right)_{\frac{1}{2}} \\
& =\left(\mathbf{f}_{h}^{n}, \frac{\mathbf{u}_{h}^{n+1}-\mathbf{u}_{h}^{n-1}}{2 \Delta t}\right)_{0} .
\end{aligned}
$$

Taking the average of Equation (A.10a) between time steps $t^{n-\frac{1}{2}}$ and $t^{n+\frac{1}{2}}$ gives:

$$
\begin{gathered}
\frac{1+p}{4}\left[\left(\mathbf{s}_{h}^{n+1}-\mathbf{s}_{h}^{n}, \frac{\mathbf{s}_{h}^{n+1}+\mathbf{s}_{h}^{n}}{2}\right)_{\frac{1}{2}}+\left(\mathbf{s}_{h}^{n}-\mathbf{s}_{h}^{n-1}, \frac{\mathbf{s}_{h}^{n}+\mathbf{s}_{h}^{n-1}}{2}\right)_{\frac{1}{2}}\right] \\
+\frac{1-p}{2}\left[\left\|\frac{\mathbf{s}_{h}^{n+1}+\mathbf{s}_{h}^{n}}{2}\right\|_{\frac{1}{2}}^{2}+\left\|\frac{\mathbf{s}_{h}^{n}+\mathbf{s}_{h}^{n-1}}{2}\right\|_{\frac{1}{2}}^{2}\right] \\
=-\frac{H_{0} p}{2(1-p)}\left[\left(\mathbf{D}_{h}\left(\mathbf{u}_{h}^{n+1}-\mathbf{u}_{h}^{n}\right), \frac{\mathbf{s}_{h}^{n+1}+\mathbf{s}_{h}^{n}}{2}\right)_{\frac{1}{2}}\right. \\
\left.+\left(\mathbf{D}_{h}\left(\mathbf{u}_{h}^{n}-\mathbf{u}_{h}^{n-1}\right), \frac{\mathbf{s}_{h}^{n}+\mathbf{s}_{h}^{n-1}}{2}\right)_{\frac{1}{2}}\right] .
\end{gathered}
$$

Besides, noticing that

$$
\begin{aligned}
& \left(\mathbf{D}_{h}\left(\mathbf{u}_{h}^{n+1}-\mathbf{u}_{h}^{n}\right), \frac{\mathbf{s}_{h}^{n+1}+\mathbf{s}_{h}^{n}}{2}\right)_{\frac{1}{2}}+\left(\mathbf{D}_{h}\left(\mathbf{u}_{h}^{n}-\mathbf{u}_{h}^{n-1}\right), \frac{\mathbf{s}_{h}^{n}+\mathbf{s}_{h}^{n-1}}{2}\right)_{\frac{1}{2}} \\
& =\frac{1}{2}\left[\left(\mathbf{D}_{h}\left(\mathbf{u}_{h}^{n+1}-\mathbf{u}_{h}^{n}\right), \mathbf{s}_{h}^{n+1}-\mathbf{s}_{h}^{n}\right)_{\frac{1}{2}}-\left(\mathbf{D}_{h}\left(\mathbf{u}_{h}^{n}-\mathbf{u}_{h}^{n-1}\right), \mathbf{s}_{h}^{n}-\mathbf{s}_{h}^{n-1}\right)_{\frac{1}{2}}\right] \\
& \quad+\left(\mathbf{D}_{h}\left(\mathbf{u}_{h}^{n+1}-\mathbf{u}_{h}^{n-1}\right), \mathbf{s}_{h}^{n}\right)_{\frac{1}{2}},
\end{aligned}
$$

Equation (A.11) may be rewritten as a function of $\left(\mathbf{s}_{h}^{n}, \mathbf{D}_{h}\left(\frac{\mathbf{u}_{h}^{n+1}-\mathbf{u}_{h}^{n-1}}{2 \Delta t}\right)\right)_{\frac{1}{2}}$ :

$$
\begin{aligned}
& -\frac{1-p^{2}}{8 H_{0} p \Delta t}\left[\left(\mathbf{s}_{h}^{n+1}-\mathbf{s}_{h}^{n}, \mathbf{s}_{h}^{n+1}+\mathbf{s}_{h}^{n}\right)_{\frac{1}{2}}+\left(\mathbf{s}_{h}^{n}-\mathbf{s}_{h}^{n-1}, \mathbf{s}_{h}^{n}+\mathbf{s}_{h}^{n-1}\right)_{\frac{1}{2}}\right] \\
& -\frac{(1-p)^{2}}{2 H_{0} p \Delta t}\left[\left\|\frac{\mathbf{s}_{h}^{n+1}+\mathbf{s}_{h}^{n}}{2}\right\|_{\frac{1}{2}}^{2}+\left\|\frac{\mathbf{s}_{h}^{n}+\mathbf{s}_{h}^{n-1}}{2}\right\|_{\frac{1}{2}}^{2}\right] \\
& -\frac{1}{4 \Delta t}\left[\left(\mathbf{D}_{h}\left(\mathbf{u}_{h}^{n+1}-\mathbf{u}_{h}^{n}\right), \mathbf{s}_{h}^{n+1}-\mathbf{s}_{h}^{n}\right)_{\frac{1}{2}}-\left(\mathbf{D}_{h}\left(\mathbf{u}_{h}^{n}-\mathbf{u}_{h}^{n-1}\right), \mathbf{s}_{h}^{n}-\mathbf{s}_{h}^{n-1}\right)_{\frac{1}{2}}\right] \\
& \quad=\left(\mathbf{s}_{h}^{n}, \mathbf{D}_{h}\left(\frac{\mathbf{u}_{h}^{n+1}-\mathbf{u}_{h}^{n-1}}{2 \Delta t}\right)\right)_{\frac{1}{2}} .
\end{aligned}
$$


The last step consists in substituting Eq. (A.12) into Eq. (A.10b). This yields to the energetic identity associated to the one-pole system (A.7):

$$
\frac{E_{h}^{n+\frac{1}{2}}-E_{h}^{n-\frac{1}{2}}}{\Delta t}=P_{h}^{n}-D_{h}^{n},
$$

where $P_{h}^{n}$ and $D_{h}^{n}$ are expressed as:

$$
\begin{aligned}
P_{h}^{n} & =\left(\mathbf{f}_{h}^{n}, \frac{\mathbf{u}_{h}^{n+1}-\mathbf{u}_{h}^{n-1}}{2 \Delta t}\right)_{0}, \\
D_{h}^{n} & =-\frac{(1-p)^{2}}{2 \Delta t H_{0} p}\left[\left\|\frac{\mathbf{s}_{h}^{n+1}+\mathbf{s}_{h}^{n}}{2}\right\|_{\frac{1}{2}}^{2}+\left\|\frac{\mathbf{s}_{h}^{n}+\mathbf{s}_{h}^{n-1}}{2}\right\|_{\frac{1}{2}}^{2}\right],
\end{aligned}
$$

and the discrete energy $E^{n+\frac{1}{2}}$ at time step $t^{n+\frac{1}{2}}$ is now given by:

$$
\begin{aligned}
E_{h}^{n+\frac{1}{2}}=\frac{\rho}{2} & \left\|\frac{\mathbf{u}_{h}^{n+1}-\mathbf{u}_{h}^{n}}{\Delta t}\right\|_{0}^{2} \\
& +\frac{1}{2} \frac{H_{0}}{1-p}\left(\mathbf{D}_{h} \mathbf{u}_{h}^{n+1}, \mathbf{D}_{h} \mathbf{u}_{h}^{n}\right)_{\frac{1}{2}}-\frac{1-p^{2}}{8 H_{0} p}\left(\left\|\mathbf{s}_{h}^{n+1}\right\|_{\frac{1}{2}}^{2}+\left\|\mathbf{s}_{h}^{n}\right\|_{\frac{1}{2}}^{2}\right) \\
& -\frac{\Delta t^{2}}{4}\left(\frac{\mathbf{s}_{h}^{n+1}-\mathbf{s}_{h}^{n}}{\Delta t}, \mathbf{D}_{h}\left(\frac{\mathbf{u}_{h}^{n+1}-\mathbf{u}_{h}^{n}}{\Delta t}\right)\right)_{\frac{1}{2}}
\end{aligned}
$$

Let us note that the previous energetic identity now involves an additional term $D_{h}^{n}$ which stands for the dissipation associated with the discrete-time behavior (A.7a).

AppendixA.3. System with pure gain and multiple one-pole filters in parallel

The previous energy analysis detailed in AppendixA.1 and AppendixA.2 may be easily extended to the more general case corresponding to a discrete timedomain behavior given by the filter (7). Let us recall the associated discrete explicit problem as obtained in Section 4.1:

$$
\begin{aligned}
\boldsymbol{\sigma}_{h k}^{n+1} & =H_{0 k} \mathbf{D}_{h} \mathbf{u}_{h}^{n+1}+p_{k} \boldsymbol{\sigma}_{h k}^{n}, \quad k=\left\{1, \ldots, N_{f}\right\}, \\
\boldsymbol{\sigma}_{h}^{n+1} & =H_{0} \mathbf{D}_{h} \mathbf{u}_{h}^{n+1}+\sum_{k=1}^{N_{f}} \boldsymbol{\sigma}_{h k}^{n+1}, \\
\rho \frac{\mathbf{u}_{h}^{n+1}-2 \mathbf{u}_{h}^{n}+\mathbf{u}_{h}^{n-1}}{\Delta t^{2}} & =\mathbf{D}_{h}^{*} \boldsymbol{\sigma}_{h}^{n}+\mathbf{f}_{h}^{n} .
\end{aligned}
$$

Similarly to what has been done in AppendixA.2 for a one-pole filter, one starts by defining the internal variables $\mathbf{s}_{h k}^{n}$ associated to each one-pole filter $H_{k}$ :

$$
\mathbf{s}_{h k}^{n}=\boldsymbol{\sigma}_{h k}^{n}-\frac{H_{0 k}}{1-p_{k}} \mathbf{D}_{h} \mathbf{u}_{h}^{n}, \quad k=\left\{1, \ldots, N_{f}\right\},
$$


such that System (A.15) becomes:

$$
\begin{aligned}
\frac{1-p_{k}}{2}\left(\mathbf{s}_{h k}^{n+1}+\mathbf{s}_{h k}^{n}\right)+ & \frac{1+p_{k}}{2}\left(\mathbf{s}_{h k}^{n+1}-\mathbf{s}_{h k}^{n}\right) \\
& =-\frac{H_{0 k} p_{k}}{1-p_{k}} \mathbf{D}_{h}\left(\mathbf{u}_{h}^{n+1}-\mathbf{u}_{h}^{n}\right), \quad k=\left\{1, \ldots, N_{f}\right\}, \\
\rho \frac{\mathbf{u}_{h}^{n+1}-2 \mathbf{u}_{h}^{n}+\mathbf{u}_{h}^{n-1}}{\Delta t^{2}} & =\sum_{k=1}^{N_{f}} \mathbf{s}_{h k}^{n}+\left(H_{0}+\sum_{k=1}^{N_{f}} \frac{H_{0 k}}{1-p_{k}}\right) \mathbf{D}_{h}^{*} \mathbf{D}_{h} \mathbf{u}_{h}^{n}+\mathbf{f}_{h}^{n} .
\end{aligned}
$$

Thereafter, each Equation (A.17a) is processed independently for each $k$ in a similar way as done in AppendixA.2, while Equation (A.17b) is processed in a similar way as done in AppendixA.1.

Finally, combining all the terms gives the same type of energetic identity as (A.13):

$$
\frac{E_{h}^{n+\frac{1}{2}}-E_{h}^{n-\frac{1}{2}}}{\Delta t}=P_{h}^{n}-D_{h}^{n},
$$

where $D_{h}^{n}$ and $E^{n+\frac{1}{2}}$ now involve the sum of the terms associated to each onepole filter while $P_{h}^{n}$ remains the same as in (A.14a):

$$
\begin{aligned}
D_{h}^{n}= & -\sum_{k=1}^{N_{f}} \frac{\left(1-p_{k}\right)^{2}}{2 \Delta t H_{0 k} p_{k}}\left[\left\|\frac{\mathbf{s}_{h k}^{n+1}+\mathbf{s}_{h k}^{n}}{2}\right\|_{\frac{1}{2}}^{2}+\left\|\frac{\mathbf{s}_{h k}^{n}+\mathbf{s}_{h k}^{n-1}}{2}\right\|_{\frac{1}{2}}^{2}\right] \\
E_{h}^{n+\frac{1}{2}}= & \frac{\rho}{2}\left\|\frac{\mathbf{u}_{h}^{n+1}-\mathbf{u}_{h}^{n}}{\Delta t}\right\|_{0}^{2}+\frac{1}{2}\left(H_{0}+\sum_{k=1}^{N_{f}} \frac{H_{0 k}}{1-p_{k}}\right)\left(\mathbf{D}_{h} \mathbf{u}_{h}^{n+1}, \mathbf{D}_{h} \mathbf{u}_{h}^{n}\right)_{\frac{1}{2}} \\
& -\sum_{k=1}^{N_{f}} \frac{1-p_{k}^{2}}{8 H_{0 k} p_{k}}\left(\left\|\mathbf{s}_{h k}^{n+1}\right\|_{\frac{1}{2}}^{2}+\left\|\mathbf{s}_{h k}^{n}\right\|_{\frac{1}{2}}^{2}\right) \\
& -\frac{\Delta t^{2}}{4} \sum_{k=1}^{N_{f}}\left(\frac{\mathbf{s}_{h k}^{n+1}-\mathbf{s}_{h k}^{n}}{\Delta t}, \mathbf{D}_{h}\left(\frac{\mathbf{u}_{h}^{n+1}-\mathbf{u}_{h}^{n}}{\Delta t}\right)\right)_{\frac{1}{2}}
\end{aligned}
$$

\section{AppendixA.4. Stability analysis}

In order to perform the stability analysis of the scheme (25), we study the conditions on the filter coefficients and discretization parameters which ensure that the discrete energy $E_{h}^{n+\frac{1}{2}}$ and dissipation $D_{h}^{n}$ in Equation (A.13) remain positive at each time step. Furthermore, we assume in the following that the condition (6) on the filter poles $p_{k}$ is fulfilled. 
First of all, let us rewrite Equation (A.18b) using the following identities:

$$
\begin{aligned}
& \left(\mathbf{D}_{h} \mathbf{u}_{h}^{n+1}, \mathbf{D}_{h} \mathbf{u}_{h}^{n}\right)_{\frac{1}{2}}=\frac{1}{4}\left(\left\|\mathbf{D}_{h}\left(\mathbf{u}_{h}^{n+1}+\mathbf{u}_{h}^{n}\right)\right\|_{\frac{1}{2}}^{2}-\left\|\mathbf{D}_{h}\left(\mathbf{u}_{h}^{n+1}-\mathbf{u}_{h}^{n}\right)\right\|_{\frac{1}{2}}^{2}\right), \\
& {\left[\left(\left\|\mathbf{s}_{h}^{n+1}\right\|_{\frac{1}{2}}^{2}+\left\|\mathbf{s}_{h}^{n}\right\|_{\frac{1}{2}}^{2}\right)-\left(\left\|\mathbf{s}_{h}^{n}\right\|_{\frac{1}{2}}^{2}+\left\|\mathbf{s}_{h}^{n-1}\right\|_{\frac{1}{2}}^{2}\right)\right]} \\
& \quad=\frac{1}{2}\left(\left\|\mathbf{s}_{h}^{n+1}+\mathbf{s}_{h}^{n}\right\|_{\frac{1}{2}}^{2}+\left\|\mathbf{s}_{h}^{n+1}-\mathbf{s}_{h}^{n}\right\|_{\frac{1}{2}}^{2}\right)-\frac{1}{2}\left(\left\|\mathbf{s}_{h}^{n}+\mathbf{s}_{h}^{n-1}\right\|_{\frac{1}{2}}^{2}+\left\|\mathbf{s}_{h}^{n}-\mathbf{s}_{h}^{n-1}\right\|_{\frac{1}{2}}^{2}\right) .
\end{aligned}
$$

This enables to split the discrete energy $E_{h}^{n+\frac{1}{2}}$ at time step $t^{n+\frac{1}{2}}$ in two distinct terms $E_{h 1}^{n+\frac{1}{2}}$ and $E_{h 2}^{n+\frac{1}{2}}$ :

$$
E_{h}^{n+\frac{1}{2}}=E_{h 1}^{n+\frac{1}{2}}+E_{h 2}^{n+\frac{1}{2}}
$$

which involve mean values and second order centered derivatives at $t^{n+\frac{1}{2}}$, respectively:

$$
\begin{aligned}
E_{h 1}^{n+\frac{1}{2}}= & \frac{1}{2}\left(H_{0}+\sum_{k=1}^{N_{f}} \frac{H_{0 k}}{1-p_{k}}\right)\left\|\mathbf{D}_{h}\left(\frac{\mathbf{u}_{h}^{n+1}+\mathbf{u}_{h}^{n}}{2}\right)\right\|_{\frac{1}{2}}^{2} \\
& -\sum_{k=1}^{N_{f}} \frac{1-p_{k}^{2}}{4 H_{0 k} p_{k}}\left\|\frac{\mathbf{s}_{h k}^{n+1}+\mathbf{s}_{h k}^{n}}{2}\right\|_{\frac{1}{2}}^{2}, \\
E_{h 2}^{n+\frac{1}{2}}=\frac{\rho}{2} \| & \frac{\mathbf{u}_{h}^{n+1}-\mathbf{u}_{h}^{n}}{\Delta t} \|_{0}^{2}-\frac{\Delta t^{2}}{4} \sum_{k=1}^{N_{f}}\left(\frac{\mathbf{s}_{h k}^{n+1}-\mathbf{s}_{h k}^{n}}{\Delta t}, \mathbf{D}_{h}\left(\frac{\mathbf{u}_{h}^{n+1}-\mathbf{u}_{h}^{n}}{\Delta t}\right)\right)_{\frac{1}{2}} \\
& -\frac{\Delta t^{2}}{8}\left(H_{0}+\sum_{k=1}^{N_{f}} \frac{H_{0 k}}{1-p_{k}}\right)\left\|\mathbf{D}_{h}\left(\frac{\mathbf{u}_{h}^{n+1}-\mathbf{u}_{h}^{n}}{\Delta t}\right)\right\|_{\frac{1}{2}}^{2} \\
& -\sum_{k=1}^{N_{f}} \frac{\Delta t^{2}\left(1-p_{k}^{2}\right)}{16 H_{0 k} p_{k}}\left\|\frac{\mathbf{s}_{h k}^{n+1}-\mathbf{s}_{h k}^{n}}{\Delta t}\right\|_{\frac{1}{2}}^{2}
\end{aligned}
$$

Let us note that Equation (A.19b) on $E_{h 1}^{n+\frac{1}{2}}$ may be rewritten using the Ztransform $H(z)$ of Filter (7) as:

$$
E_{h 1}^{n+\frac{1}{2}}=\frac{H(1)}{2}\left\|\mathbf{D}_{h}\left(\frac{\mathbf{u}_{h}^{n+1}+\mathbf{u}_{h}^{n}}{2}\right)\right\|_{\frac{1}{2}}^{2}-\sum_{k=1}^{N_{f}} \frac{1-p_{k}^{2}}{4 H_{0 k} p_{k}}\left\|\frac{\mathbf{s}_{h k}^{n+1}+\mathbf{s}_{h k}^{n}}{2}\right\|_{\frac{1}{2}}^{2} .
$$

Then, sufficient conditions in order to ensure the positivity of $E_{h 1}^{n+\frac{1}{2}}$ are:

$$
H(1) \geq 0 \quad \text { and } \quad H_{0 k} p_{k} \leq 0 \quad k=\left\{1, \ldots, N_{f}\right\},
$$

which also ensure the positivity of the dissipation $D_{h}^{n}$ (A.18a).

In order to study $E_{h 2}^{n+\frac{1}{2}}$, assuming that $H_{0 k}$ and $p_{k}$ satisfy the previous 
condition, we start by expanding the following inequality:

$$
\left\|\sqrt{-\frac{\left(1-p_{k}^{2}\right)}{2 H_{0 k} p_{k}}}\left(\frac{\mathbf{s}_{h k}^{n+1}-\mathbf{s}_{h k}^{n}}{\Delta t}\right)-\sqrt{-\frac{2 H_{0 k} p_{k}}{1-p_{k}^{2}}} \mathbf{D}_{h}\left(\frac{\mathbf{u}_{h}^{n+1}-\mathbf{u}_{h}^{n}}{\Delta t}\right)\right\|_{\frac{1}{2}}^{2} \geq 0,
$$

which yields when being multiplied by $\Delta t^{2} / 8$ :

$$
\begin{aligned}
-\frac{\Delta t^{2}}{16} \frac{1-p_{k}^{2}}{H_{0 k} p_{k}}\left\|\frac{\mathbf{s}_{h k}^{n+1}-\mathbf{s}_{h k}^{n}}{\Delta t}\right\|_{\frac{1}{2}}^{2} & -\frac{\Delta t^{2}}{4}\left(\frac{\mathbf{s}_{h k}^{n+1}-\mathbf{s}_{h k}^{n}}{\Delta t}, \mathbf{D}_{h}\left(\frac{\mathbf{u}_{h}^{n+1}-\mathbf{u}_{h}^{n}}{\Delta t}\right)\right)_{\frac{1}{2}} \\
& \geq \frac{\Delta t^{2}}{4} \frac{H_{0 k} p_{k}}{1-p_{k}^{2}}\left\|\mathbf{D}_{h}\left(\frac{\mathbf{u}_{h}^{n+1}-\mathbf{u}_{h}^{n}}{\Delta t}\right)\right\|_{\frac{1}{2}}^{2} .
\end{aligned}
$$

Then, substituting Equation (A.21) in Equation (A.19c) gives a first lower bound for $E_{h 2}^{n+\frac{1}{2}}$ as:

$$
E_{h 2}^{n+\frac{1}{2}} \geq \frac{\rho}{2}\left\|\frac{\mathbf{u}_{h}^{n+1}-\mathbf{u}_{h}^{n}}{\Delta t}\right\|_{0}^{2}-\frac{\Delta t^{2}}{8}\left(H_{0}+\sum_{k=1}^{N_{f}} \frac{H_{0 k}}{1+p_{k}}\right)\left\|\mathbf{D}_{h}\left(\frac{\mathbf{u}_{h}^{n+1}-\mathbf{u}_{h}^{n}}{\Delta t}\right)\right\|_{\frac{1}{2}}^{2} .
$$

Furthermore, let us note the following result for all $\mathbf{v}_{h} \in L^{2}\left(\Omega_{h, 0}\right)$ :

$$
\begin{aligned}
\left\|\mathbf{D}_{h} \mathbf{v}_{h}\right\|_{\frac{1}{2}}^{2} & =\frac{1}{h} \sum_{j=0}^{N_{x}-1}\left(\mathbf{v}_{h_{j+1}^{2}}^{2}+\mathbf{v}_{h j}^{2}-2 \mathbf{v}_{h j+1} \mathbf{v}_{h j}\right) \\
& \leq \frac{2}{h} \sum_{j=0}^{N_{x}-1}\left(\mathbf{v}_{h_{j+1}}^{2}+\mathbf{v}_{h_{j}}^{2}\right) \\
& \leq \frac{4}{h^{2}}\left\|\mathbf{v}_{h}\right\|_{0}^{2},
\end{aligned}
$$

which gives:

$$
\left\|\mathbf{D}_{h}\left(\frac{\mathbf{u}_{h}^{n+1}-\mathbf{u}_{h}^{n}}{\Delta t}\right)\right\|_{\frac{1}{2}}^{2} \leq \frac{4}{h^{2}}\left\|\frac{\mathbf{u}_{h}^{n+1}-\mathbf{u}_{h}^{n}}{\Delta t}\right\|_{0}^{2}
$$

Using this last result allows us to derive a new lower bound on $E_{h 2}^{n+\frac{1}{2}}$ as:

$$
E_{h 2}^{n+\frac{1}{2}} \geq \frac{1}{2}\left[\rho-\frac{\Delta t^{2}}{h^{2}}\left(H_{0}+\sum_{k=1}^{N_{f}} \frac{H_{0 k}}{1+p_{k}}\right)\right]\left\|\frac{\mathbf{u}_{h}^{n+1}-\mathbf{u}_{h}^{n}}{\Delta t}\right\|_{0}^{2} .
$$

Finally, a sufficient condition that ensure positivity of $E_{h 2}^{n+\frac{1}{2}}$, involving the discretization parameters $h$ and $\Delta t$ is:

$$
\rho-\frac{\Delta t^{2}}{h^{2}}\left(H_{0}+\sum_{k=1}^{N_{f}} \frac{H_{0 k}}{1+p_{k}}\right) \geq 0 .
$$


We recognizes here a classic CFL condition, which may be rewritten in a more compact way involving transfer function $H(z)$ as:

$$
\Delta t \leq h \sqrt{\frac{\rho}{H(-1)}} .
$$

\section{AppendixB. Reference modal solution}

This section describes the modal analysis performed in order to obtain the reference solution used for validating our numerical results in Section 5.3. To do so, we start again from the equation setting of our model problem and look for an analytical solution in separated variables form:

$$
u(x, t)=X(x) T(t)
$$

First of all, we apply the partial time-domain Fourier transform to Equation (1), where the volumetric force density has been chosen as $f(x, t)=F(t) \delta(x-$ $L)=F(t) \delta_{L}(x)$ as in Section 5. Using the linear and local property of the constitutive law $\mathcal{E}$ yields:

$$
-\rho \omega^{2} \hat{u}(x, \omega)-E^{*}(\omega) \frac{\partial^{2} \hat{u}(x, \omega)}{\partial x^{2}}=\delta_{L}(x) \hat{F}(\omega),
$$

where $E^{*}(\omega)$ is the frequency-domain response of operator $\mathcal{E}, \hat{F}(\omega)$ is the Fourier transform of $F(t)$ and $\hat{u}(x, \omega)$ writes $\hat{u}(x, \omega)=X(x) \hat{T}(\omega)$.

Next, substituting the separated form (B.1) in the homogeneous counterpart of Equation (B.2) gives the classical eigenvalue problem in terms of the space function $X$ :

$$
\frac{\partial^{2} X(x)}{\partial x^{2}}+k^{2} X(x)=0 .
$$

Solving the previous problem together with the choice of clamped-free boundary conditions given in Equation (2.1) allows us to express the following orthonormalized family of functions $\left\{X_{n}\right\}_{n \in \mathbb{N}^{*}}$ which is the modal basis of Problem (1):

$$
X_{n}(x)=\sqrt{\frac{2}{L}} \sin \left(k_{n} x\right) \quad \text { with } \quad k_{n}=\left(n-\frac{1}{2}\right) \frac{\pi}{L} \quad\left(n \in \mathbb{N}^{*}\right),
$$

where the usual $L^{2}$ norm $\left\|X_{n}\right\|$ has been used for normalization:

$$
\left\|X_{n}\right\|=\left[\int_{0}^{L} X_{n}(x)^{2} d x\right]^{\frac{1}{2}} .
$$


Let us recall the fundamental properties of family $\left\{X_{n}\right\}_{n \in \mathbb{N}^{*}}$ :

$$
\left(X_{n}, X_{m}\right)=\delta_{n, m} \quad \text { and } \quad \frac{\partial^{2} X_{n}(x)}{\partial x^{2}}=-k_{n}^{2} X_{n}(x),
$$

where $\left(X_{n}, X_{m}\right)$ denotes the usual $L^{2}$ scalar product:

$$
\left(X_{n}, X_{m}\right)=\int_{0}^{L} X_{n}(x) X_{m}(x) d x .
$$

We now express the solution $\hat{u}(x, \omega)$ of the space-frequency-domain problem on Basis (B.3):

$$
\hat{u}(x, \omega)=\sum_{n=1}^{\infty} X_{n}(x) \hat{T}_{n}(\omega),
$$

and substitute this expression into Equation (B.2):

$$
\sum_{n=1}^{\infty}\left(-\rho \omega^{2}+k_{n}^{2} E^{*}(\omega)\right) X_{n}(x) \hat{T}_{n}(\omega)=\delta_{L}(x) \hat{F}(\omega) .
$$

Next, the orthogonality property allows us to express $\hat{T}_{n}(\omega)$ as:

$$
\hat{T}_{n}(\omega)=\frac{\hat{F}(\omega)}{-\rho \omega^{2}+k_{n}^{2} E^{*}(\omega)} \frac{\left(\delta_{L}, X_{n}\right)}{\left\|X_{n}\right\|^{2}} .
$$

Noting that $\left\|X_{n}\right\|^{2}=1$ and $\left(\delta_{L}, X_{n}\right)=X_{n}(L)$, the frequency-domain analytical solution of model Problem (1) is given as follows:

$$
\hat{u}(x, \omega)=\sum_{n=1}^{\infty} \frac{\hat{F}(\omega)}{-\rho \omega^{2}+k_{n}^{2} E^{*}(\omega)} X_{n}(L) X_{n}(x) .
$$

Finally, in order to compute the reference modal solution used to compare the numerical results of Section 5.3, we set $E^{*}(\omega)=H^{c}(\omega)$ and truncate the infinite sum of Equation (B.5) to 212 modes, which corresponds to all the modes up to $180 \mathrm{kHz}$. 